\title{
Observer based region tracking control for underwater vehicles without velocity measurement
}

\author{
Xing Liu · Mingjun Zhang · Feng Yao · Zhenzhong Chu
}

Received: date / Accepted: date

\begin{abstract}
This paper addresses the problem of region tracking control for underwater vehicles without velocity measurement in marine environment. For this case, an improved region tracking control strategy is proposed based on a Nussbaum state observer. In the proposed method, a Nussbaum state observer is developed to estimate the unmeasured velocity of the vehicle. And then an improved region tracking control strategy is presented by incorporating the estimated results of the state observer, such that the tracking errors satisfy the requirement of the prescribed boundaries. In addition, a RBF neural network is applied to approximate the unknown dynamics of the vehicle. It is verified that the estimated error and the tracking error are uniformly ultimately bounded. Finally, the proposed observer-based region tracking control strategy is applied on an underwater vehicle to perform simulation studies and compared with a traditional backstepping controller and a traditional region tracking controller based on a high-gain observer. The comparative simulation results demonstrate the effectiveness of the proposed region tracking control strategy.
\end{abstract}

Keywords Underwater vehicles · region tracking control $\cdot$ state observer $\cdot$ Nussbaum function

Xing Liu (Corresponding Author), Mingjun Zhang, Feng Yao College of Mechanical and Electrical Engineering, Harbin Engineering University, Harbin, 150001, China.

E-mail: liuxing20080724@gmail.com

Zhenzhong Chu

Shanghai Engineering Research Center of Intelligent Maritime Search \& Rescue and Underwater Vehicles, Shanghai Maritime University, Shanghai 201306, People's Republic of China.

\section{Introduction}

Due to their special advantages, e.g., moving in complex marine environment, especially in deep-sea environment, underwater vehicles have wide applications, including underwater detection, underwater searching, and operation with manipulators $[1-4$. Among these applications, a reliable control system is required for successful completion of the given mission 58 . Due to the existence of ocean current, modelling uncertainty and nonlinear coupling, there are many difficulties in the control design of underwater vehicles, which is also a current research hot 911 . In control strategies of underwater vehicles, the concept of region tracking control has been proposed by considering the speciality of some applications [12], e.g., the tracking precision is not their first priority in pipeline tracking. And for these special missions, the control signals are expected to be as smooth as possible on the premise that the tracking errors of the vehicle are always kept within the prescribed boundaries. It is also the original intention of region tracking control.

From the existing region tracking control schemes, most of them only focus on steady performance of the tracking error 13 16, i.e., the region tracking is achieved if the tracking error of the vehicle in the steady state is within the prescribed boundaries. As a representative of the design previously reported, [12] proposed a potential energy function based region tracking control scheme, where the tracking errors converged to the desired region in the steady state. Another representative is the region tracking control scheme based on the piecewise and differential Lyapunov function in [17], where the region tracking control law was derived in the framework of backstepping technique by the special Lyapunov functions with the desired region. However, if 
the requirement about the transient-state performance of the tracking error is made in some special missions, the aforementioned region tracking control schemes are not applicable.

Recently, prescribed performance control (PPC) has been proposed, where the tracking errors can satisfy the prescribed requirements in both the transient state and the steady state by the constraint transformation 18 20. The PPC's idea maybe provide a choice for the design of region tracking control with the added requirements in the transient state. However, from the simulation results of the existing PPC schemes, the tracking error always converges to zero, which does not satisfy the original intention of region tracking control. To solve this problem, 21 proposed an adaptive region tracking control with the prescribed transient performance for underwater vehicles, where a error transformation inspired by PPC was combined with the the piecewise and differential Lyapunov function to design the region tracking control law.

It is worth noting that both position and velocity of the vehicle should be available in the existing region tracking control schemes. However, the sensors carried by an underwater vehicle sometimes cannot provide such enough state information for the control system, due to the cost or other practical issues 22, 23. For example, the Doppler velocity log (DVL), always used to measure the velocity of the vehicle, requires that the vehicle's attitude off the seafloor is less than a maximum range which is related with the DVL's frequency and DVL is also susceptible to sporadic failures [24. For this scenario where the velocity of the vehicle is not available, it is necessary to investigate the region tracking control problem for underwater vehicles with only position measurement.

For the control design without velocity measurement, observer-based control strategies have been considered to implement tracking control for underwater vehicles only equipped with position sensors $9,23,25$, but which have been investigated for tracking control system with high precision. It is known that state observers have the ability to well estimate the unmeasured state by using the known state information of the vehicle and its control input. So far, different forms of state observers have been presented. Among them, high-gain observer or variants thereof has been widely applied to estimate the unmeasured velocity, due to its simple structure 26,27]. In order to obtain more precise estimation results, other state observers with complex forms have been investigated. For example, adaptive sliding mode based observers were developed in 22, 23, where the finite-time convergence of the estimated errors was achieved and the estimation precision was im- proved. According to the existing forms of state observers, it is known that the estimation ability about the unmeasured state can be enhanced by introducing nonlinear feedback in the state observers. Hence, this paper develops a new form of state observer by introducing Nussbaum-type functions.

Nussbaum-type functions have an important property for uniformly ultimately boundedness. For the compounded disturbance term in the general MIMO system, 28 proposed a Nussbaum disturbance observer based on the complete states. On this basis, 29] gives an improved Nussbaum disturbance observer based on RBF neural network. These Nussbaum-type observers in 28,29 have shown good estimation precision for the general disturbances. However, they all require the full states of the nonlinear systems, which means that these forms of the Nussbaum-type observers cannot be directly used to estimate the unmeasured state of the nonlinear system.

Motivated by the aforementioned discussions, this paper investigates observer-based region tracking control problem for underwater vehicles with only position measurement. In this paper, the unknown dynamics is on-line approximated by a RBF neural network. The objective of this paper is to estimate the unmeasured velocity of the vehicle and guarantee the tracking error to be kept within the prescribed boundaries in the whole process. The main contributions of this paper are summarized as follows:

(1) An observer-based region tracking control strategy is proposed for underwater vehicles with only position measurement. To our best knowledge, in the existing region tracking control schemes, both position sensors and velocity sensors are required.

(2) A Nussbaum-type state observer is introduced to estimate the unmeasured velocities. And to achieve region tracking control and reduce the chattering phenomenon of the control inputs, an improved region tracking control strategy is developed. Specifically, a new error transformation is introduced into the concept of prescribed performance control to make the tracking error to be enlarged but still always kept within the prescribed boundaries.

The rest of this paper is organized as follows. Problem statement, including the dynamic model of an underwater vehicle, is presented in Section 2. Section 3 focuses on the designs of a Nussbaum-type state observer and an improved region tracking controller. In Section 4, comparative simulation results are provided to demonstrate the effectiveness of the proposed observerbased region tracking control strategy. Finally, conclusions are draw in Section 5 . 


\section{Problem statement}

2.1 Underwater vehicles' dynamics

Consider the dynamics of an underwater vehicle with six degrees of freedom (DOFs) fully actuated by thrusters 7, 30, given by

$$
\begin{aligned}
\ddot{\eta}= & M_{\eta}^{-1}(\eta) J B u-M_{\eta}^{-1}(\eta)\left(C_{R B \eta}(\dot{\eta}) \dot{\eta}\right) \\
& -M_{\eta}^{-1}(\eta)\left(C_{A \eta}\left(\dot{\eta}_{r}\right) \dot{\eta}_{r}+D_{\eta}\left(\dot{\eta}_{r}\right) \dot{\eta}_{r}+G_{\eta}(\eta)\right)
\end{aligned}
$$

where $\eta \in \mathbb{R}^{6}$ is the position and attitude vector, $M_{\eta}(\eta) \in$ $\mathbb{R}^{6 \times 6}$ denotes the inertia matrix including the added matrix, $C_{R B \eta}(\dot{\eta}) \in \mathbb{R}^{6 \times 6}$ expresses the rigid-body centripetal and Coriolis matrix, $C_{A \eta}\left(\dot{\eta}_{r}\right) \in \mathbb{R}^{6 \times 6}$ shows the hydrodynamic centripetal and Coriolis matrix, $D_{\eta}\left(\dot{\eta}_{r}\right) \in$ $\mathbb{R}^{6 \times 6}$ is the hydrodynamics damping matrix, $G_{\eta}(\eta) \in$ $\mathbb{R}^{6}$ is the gravity and buoyancy vector. $\dot{\eta}_{r}=\dot{\eta}-\dot{\eta}_{c}, \dot{\eta}_{c}$ is the speed of ocean current. $J \in \mathbb{R}^{6 \times 6}$ is the velocity transformation matrix from the body-fixed frame to the earth inertial frame. $B \in \mathbb{R}^{6 \times n}$ is the thruster distribution matrix, $n \geq 6$ denotes the number of thrusters, $u \in \mathbb{R}^{n}$ is the control input of thrusters.

From Eq.(1), the dynamics of an underwater vehicle is rewritten to simplify the control design, shown as

$\ddot{\eta}=M_{\eta}^{-1}(\eta) J B u+F(\eta, \dot{\eta})$

where $F(\eta, \dot{\eta})=-M_{\eta}^{-1}(\eta)\left(C_{R B \eta}(\dot{\eta}) \dot{\eta}+C_{A \eta}\left(\dot{\eta}_{r}\right) \dot{\eta}_{r}\right)-$ $M_{\eta}^{-1}(\eta)\left(D_{\eta}\left(\dot{\eta}_{r}\right) \dot{\eta}_{r}+G_{\eta}(\eta)\right)$ will be estimated by a neural network in the next section.

Let $\bar{x}_{1}=\eta \in \mathbb{R}^{6}, \bar{x}_{2}=\dot{\eta} \in \mathbb{R}^{6}$, and define $\bar{x}=$ $\left[\bar{x}_{1}^{T}, \bar{x}_{2}^{T}\right]^{T}$. Also set $x=\left[x_{1}^{T}, x_{2}^{T}\right]^{T}$ with $x_{1}, x_{2} \in \mathbb{R}^{6}$. Then, introduce the coordinate transformation

$x=\left[\begin{array}{cc}I_{6 \times 6} & 0_{6 \times 6} \\ -T_{1} & I_{6 \times 6}\end{array}\right] \bar{x}$

where $I_{6 \times 6}$ is a six-order identity matrix while $0_{6 \times 6}$ is a six-order null matrix. $T_{1} \in \mathbb{R}^{6 \times 6}$ is a positive-definite diagonal matrix. form

Eq. (2) can be rewritten as the following state-space

$\dot{x}_{1}=T_{1} x_{1}+x_{2}$

$\dot{x}_{2}=-T_{1}^{2} x_{1}-T_{1} x_{2}+\bar{F}\left(x_{1}, x_{2}\right)+E\left(x_{1}\right) u$,

where $\bar{F}\left(x_{1}, x_{2}\right)=F(\eta, \dot{\eta})$ and $E\left(x_{1}\right)=M_{\eta}^{-1}(\eta) J B$.
A continuous function $N(\bullet)$ is considered as a Nussbaumtype function if it has the following prosperities 31,32 :

$\lim _{k \rightarrow+\infty} \sup \frac{1}{k} \int_{0}^{k} N(\chi) d \chi=+\infty$

$\lim _{k \rightarrow+\infty} \inf \frac{1}{k} \int_{0}^{k} N(\chi) d \chi=-\infty$

Typically, the continuous function $k^{2} \cos (k), k^{2} \sin (k)$ are selected as Nussbaum-type functions.

Lemma 1 31, 32] Let $V(t), \varsigma_{i}(t), i=1,2, \ldots, N$ be smooth functions defined on $\left[0, t_{f}\right]$ with $V(t) \geq 0$ and $\varsigma_{i}(0)=0$. And $N(\bullet)$ is Nussbaum type functions. If the following inequality holds:

$V(t) \leq C+\exp \left(-c_{a} t\right) \sum_{i=1}^{N} \int_{0}^{t}(\Xi(\tau) d \tau)$,

where $\Xi(\tau)=\left(g(\tau) N\left(\varsigma_{i}(\tau)\right)-1\right) \dot{\varsigma}_{i}(\tau) \exp \left(c_{a} \tau\right) . C$ and $c_{a}$ are positive constants. $g(t)$ is a time-varying parameter that takes values in the unknown set $I=$ $\left[g^{-}, g^{+}\right]$with $0 \notin I$, then $V(t), \varsigma_{i}(t), i=1,2, \ldots, N$, $\sum_{i=1}^{N} \int_{0}^{t}\left(g(\tau) N\left(\varsigma_{i}(\tau)\right)-1\right) \dot{\varsigma}_{i}(\tau) \exp \left(c_{a} \tau\right) d \tau$ are bounded on $\left[0, t_{f}\right]$.

\subsection{Control objective}

Considering special tasks of underwater vehicles, e.g., pipeline tracking or target searching, high control precision sometimes is not their first priority, while longer operation time and smoother control signals may be pursued, after all the cost of implementation for one task is much expensive. In addition, when implementing the state-feedback controller to underwater vehicles, the position/attitude $\eta$ of an underwater vehicle can be measured and provided by position sensors, such as ultrashort baseline and compass module. However, due to the cost and other practical reasons, as discussed in Section 1, the velocity $\dot{\eta}$ cannot be always available. In this scenario, this paper investigates the region tracking control problem for underwater vehicles without velocity measurement. The objective of this paper is twofold: 1) to design a state observer to estimate the unmeasurable velocity $\dot{\eta}$ and 2) to derive a region tracking control strategy such that the tracking errors are always kept within the prescribed boundaries but do not converge to zero. 


\section{Observer based region tracking control}

In this section, an adaptive region tracking control scheme is proposed based on a Nussbaum-type state observer for underwater vehicles in presence of ocean current disturbances and unmeasured velocity state. The overall conceptual diagram of the proposed observer-based region tracking control strategy is presented in Fig. 1.

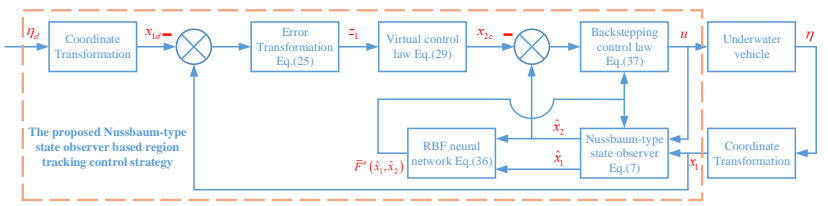

Fig. 1 The overall conceptual diagram of the proposed observer-based region tracking control strategy

\subsection{Nussbaum state observer}

To estimate the unmeasured state $x_{2}$ in (4), the Nussbaum state observer is designed as

$$
\begin{aligned}
& \dot{\hat{x}}_{1}=T_{1} \hat{x}_{1}+\hat{x}_{2}+L_{1} \Delta_{1}+f_{1}\left(\Delta_{1}\right) \\
& \dot{\hat{x}}_{2}=-T_{1}^{2} \hat{x}_{1}-T_{1} \hat{x}_{2}+\bar{F}^{e}\left(\hat{x}_{1}, \hat{x}_{2}\right)+E\left(x_{1}\right) u \\
& \quad+L_{2} \Delta_{1}+f_{2}\left(\Delta_{1}\right)
\end{aligned}
$$

where $\Delta_{1}=x_{1}-\hat{x}_{1}, L_{1}$ and $L_{2}$ are diagonal matrixes, $\bar{F}^{e}\left(\hat{x}_{1}, \hat{x}_{2}\right)$ is the output of a neural network given in the next subsection, and it is always assumed that 23, 33.

$$
\left\|\bar{F}\left(x_{1}, x_{2}\right)-\bar{F}^{e}\left(\hat{x}_{1}, \hat{x}_{2}\right)\right\| \leq \delta\left\|x_{2}-\hat{x}_{2}\right\|,
$$

where $\delta$ is a known positive constant, and $\|\cdot\|$ denote the 2 -norm of a vector. Then, $f_{1}\left(\Delta_{1}\right)$ and $f_{2}\left(\Delta_{1}\right)$ are given by

$$
\begin{aligned}
f_{1}\left(\Delta_{1}\right) & =k_{0} \int_{0}^{t}\left\lfloor\Delta_{1}\right\rfloor^{\alpha} d \tau+k_{1}\left\lfloor\Delta_{1}\right\rfloor^{\gamma_{1}}+k_{2} \Delta_{1} \\
& +k_{3}\left\lfloor\Delta_{1}\right\rfloor^{\gamma_{2}}-p_{1} \operatorname{col}\left(\left(a_{1} N_{1 i}\left(\xi_{1 i}\right)-1\right)\right) \\
f_{2}\left(\Delta_{1}\right) & =\left(T_{1}^{2}+I_{6 \times 6}\right) P_{1} P_{2}^{-1}\left\lfloor\Delta_{1}\right\rfloor^{\alpha}+k_{5} \operatorname{sign}\left(\Delta_{1}\right) \\
& -p_{2} \operatorname{col}\left(\left(a_{2} N_{2 i}\left(\xi_{2 i}\right)-1\right)\right),
\end{aligned}
$$

where $k_{j}(j=0,1, \ldots, 6), p_{1}, p_{2}, a_{1}$ and $a_{2}$ are positive constants, $\gamma_{1} \in(0,1), \gamma_{2}>1, \alpha \in(0,1) . P_{1}$ and $P_{2}$ are positive-definite diagonal matrixes. $\left\lfloor\Delta_{1}\right\rfloor^{\gamma_{1}}=$ $\left[\left|\Delta_{11}\right|^{\gamma_{1}} \operatorname{sign}\left(\Delta_{11}\right), \ldots,\left|\Delta_{16}\right|^{\gamma_{1}} \operatorname{sign}\left(\Delta_{16}\right)\right]^{T}, \Delta_{1 i}$ is the $i$ th entry of $\Delta_{1} \cdot\left\lfloor\Delta_{1}\right\rfloor^{\alpha}$ and $\left\lfloor\Delta_{1}\right\rfloor^{\gamma_{2}}$ have the similar forms as $\left\lfloor\Delta_{1}\right\rfloor^{\gamma_{1}}$. The operation "col" means to combine elements to be a column vector and $i=1, \ldots, 6$. $\xi_{1 i}$ and $\xi_{2 i}$ are the $i$ th entry of vectors $\xi_{1}$ and $\xi_{2}$, respectively. $N_{1 i}\left(\xi_{1 i}\right)$ and $N_{2 i}\left(\xi_{2 i}\right)$ are Nussbaum-type functions.

And

$$
\begin{aligned}
& \xi_{1}=k_{4} \int_{0}^{t}\left\lfloor\Delta_{1}\right\rfloor^{\alpha} d \tau \\
& \xi_{2}=k_{6}\left(\Delta_{1}+\int_{0}^{t}\left(L_{1} \Delta_{1}-T_{1} \Delta_{1}+f_{1}\left(\Delta_{1}\right)\right) d \tau\right) .
\end{aligned}
$$

Lemma 2 Consider underwater vehicles described as (1), and suppose the assumption (8) holds. After using the coordinate transformation (3) and applying the designed Nussbaum-type state observer (7) to estimate the unmeasured velocity, if the following inequality holds

$Q=P A_{0}+A_{0}^{T} P+\frac{2}{\varepsilon_{0}} P P+\Psi<0$,

where $\Psi=\operatorname{diag}\left(0_{6 \times 6}, \varepsilon_{0} \delta^{2} I_{6 \times 6}\right), A_{0}=A-\left[\begin{array}{cc}L_{1} & 0_{6 \times 6} \\ L_{2} & 0_{6 \times 6}\end{array}\right]$, $A=\left[\begin{array}{cc}T_{1} & I_{6 \times 6} \\ -T_{1}^{2} & -T_{1}\end{array}\right]$, and $P=\operatorname{diag}\left(P_{1}, P_{2}\right)$.

then the estimated error is guaranteed to be uniformly ultimately bounded.

Proof The following error dynamic equation is obtained by subtracting (7) from (4), given by

$$
\begin{aligned}
\dot{\Delta}_{1} & =\left(T_{1}-L_{1}\right) \Delta_{1}+\Delta_{2}-f_{1}\left(\Delta_{1}\right) \\
\dot{\Delta}_{2} & =-T_{1}^{2} \Delta_{1}-L_{2} \Delta_{1}-T_{1} \Delta_{2}+\bar{F}\left(x_{1}, x_{2}\right) \\
& -\bar{F}^{e}\left(\hat{x}_{1}, \hat{x}_{2}\right)-f_{2}\left(\Delta_{1}\right),
\end{aligned}
$$

where $\Delta_{2}=x_{2}-\hat{x}_{2}$.

Consider the following Lyapunov function

$$
\begin{aligned}
V_{1} & =k_{0}\left[\int_{0}^{t}\left\lfloor\Delta_{1}\right\rfloor^{\alpha} d \tau\right]^{T} P_{1} \int_{0}^{t}\left\lfloor\Delta_{1}\right\rfloor^{\alpha} d \tau \\
& +\frac{2}{1+\alpha}\left[\left\lfloor\Delta_{1}\right\rfloor^{\alpha}\right]^{T} P_{1} \Delta_{1}+\Delta_{2}^{T} P_{2} \Delta_{2} .
\end{aligned}
$$

Since $P_{1}$ and $P_{2}$ are positive-definite diagonal matrixes, it has the following equation:

$$
\begin{aligned}
{\left[\left\lfloor\Delta_{1}\right\rfloor^{\alpha}\right]^{T} P_{1} \Delta_{1} } & =\sum_{i=1}^{6}\left(\left|\Delta_{1 i}\right|^{\alpha} \operatorname{sign}\left(\Delta_{1 i}\right) P_{1 i} \Delta_{1 i}\right) \\
& =\sum_{i=1}^{6}\left(P_{1 i}\left|\Delta_{1 i}\right|^{\alpha+1}\right),
\end{aligned}
$$


where $\Delta_{1 i}$ is the $i$ th element of $\Delta_{1}$; and $P_{1 i}$ is the $i$ th diagonal element of the diagonal matrix $P_{1}$. Hence, $\left[\left\lfloor\Delta_{1}\right\rfloor^{\alpha}\right]^{T} P_{1} \Delta_{1}$ is nonnegative. Similarly, it also has that $\left[\int_{0}^{t}\left\lfloor\Delta_{1}\right\rfloor^{\alpha} d \tau\right]^{T} P_{1} \int_{0}^{t}\left\lfloor\Delta_{1}\right\rfloor^{\alpha} d \tau$ is nonnegative. Therefore, it can be concluded that the Lyapunov function $V_{1}$ is always nonnegative for any $t \geq 0$.

From Eq. [14, the derivative of $\left[\left\lfloor\Delta_{1}\right\rfloor^{\alpha}\right]^{T} P_{1} \Delta_{1}$ with respect to time can be described as follows:

$$
\begin{aligned}
\frac{d}{d t}\left(\left[\left\lfloor\Delta_{1}\right\rfloor^{\alpha}\right]^{T} P_{1} \Delta_{1}\right) & =\frac{d}{d t}\left(\sum_{i=1}^{6}\left(P_{1 i}\left|\Delta_{1 i}\right|^{\alpha+1}\right)\right) \\
& =(\alpha+1) \sum_{i=1}^{6} P_{1 i}\left|\Delta_{1 i}\right|^{\alpha} \operatorname{sign}\left(\Delta_{1 i}\right) \dot{\Delta}_{1 i} \\
& =(\alpha+1)\left[\left\lfloor\Delta_{1}\right\rfloor^{\alpha}\right]^{T} P_{1} \dot{\Delta}_{1}
\end{aligned}
$$

Similarly, since $P_{2}$ is the diagonal matrix, it follows that $\Delta_{2}^{T} P_{2} \dot{\Delta}_{2}=\dot{\Delta}_{2}^{T} P_{2} \Delta_{2}$.

Then the time derivative of $V_{1}$ is given by

$$
\begin{aligned}
\dot{V}_{1} & =2 k_{0}\left[\int_{0}^{t}\left\lfloor\Delta_{1}\right\rfloor^{\alpha} d \tau\right]^{T} P_{1}\left\lfloor\Delta_{1}\right\rfloor^{\alpha}+2\left[\left\lfloor\Delta_{1}\right\rfloor^{\alpha}\right]^{T} P_{1} \dot{\Delta}_{1} \\
& +2 \Delta_{2}^{T} P_{2} \dot{\Delta}_{2}
\end{aligned}
$$

Let $X=\operatorname{col}\left(\left\lfloor\Delta_{1}\right\rfloor^{\alpha}, \Delta_{2}\right)$, and substitute 12 into (16). It follows that

$$
\begin{aligned}
\dot{V}_{1} & =\Delta^{T} A_{0}^{T} P X+2\left[\left\lfloor\Delta_{1}\right\rfloor^{\alpha}\right]^{T} P_{1}\left(T_{1}^{2}+I_{6 \times 6}\right) \Delta_{2} \\
& +X^{T} P A_{0} \Delta+2 \Delta_{2}^{T} P_{2}\left(\bar{F}\left(x_{1}, x_{2}\right)-\bar{F}^{e}\left(x_{1}, x_{2}\right)\right) \\
& -2\left[\left\lfloor\Delta_{1}\right\rfloor^{\alpha}\right]^{T} P_{1}\left(k_{1}\left\lfloor\Delta_{1}\right\rfloor^{\gamma_{1}}+k_{2} \Delta_{1}\right) \\
& -2\left[\left\lfloor\Delta_{1}\right\rfloor^{\alpha}\right]^{T} P_{1}\left(k_{3}\left\lfloor\Delta_{1}\right\rfloor^{\gamma_{2}}-p_{1} \operatorname{col}\left(\left(a_{1} N_{1 i}\left(\xi_{1 i}\right)-1\right)\right)\right) \\
& -2 \Delta_{2}^{T} P_{2}\left(\left(T_{1}^{2}+I_{6}\right) P_{1} P_{2}^{-1}\left\lfloor\Delta_{1}\right\rfloor^{\alpha}+k_{5} \operatorname{sign}\left(\Delta_{1}\right)\right) \\
& +2 p_{2} \Delta_{2}^{T} P_{2} \operatorname{col}\left(\left(a_{2} N_{2 i}\left(\xi_{2 i}\right)-1\right)\right) .
\end{aligned}
$$

According to the Young's inequality 34] and the assumption (8), it follows that

$$
\begin{aligned}
2 \Delta_{2}^{T} P_{2}\left(\bar{F}\left(x_{1}, x_{2}\right)-\bar{F}^{e}\left(\hat{x}_{1}, \hat{x}_{2}\right)\right) & \leq \frac{1}{\varepsilon_{0}} X^{T} P P \Delta \\
& +\varepsilon_{0} \delta^{2} \Delta_{2}^{T} \Delta_{2},
\end{aligned}
$$

where $\varepsilon_{0}$ is a positive constant.

Similarly, the following inequality is also obtained based on the Young's inequality 34 .

$$
\begin{aligned}
2 \Delta_{2}^{T} P_{2} k_{5} \operatorname{sign}\left(\Delta_{1}\right) & \leq \frac{1}{\varepsilon_{0}} \Delta_{2}^{T} P_{2} P_{2} \Delta_{2}+\varepsilon_{0} k_{5}^{2} \\
& \leq \frac{1}{\varepsilon_{0}} X^{T} P P \Delta+\varepsilon_{0} k_{5}^{2} .
\end{aligned}
$$

Substituting (18) and (19) into 17, we have

$$
\begin{aligned}
\dot{V}_{1} & \leq X^{T} P A_{0} \Delta+\Delta^{T} A_{0}^{T} P X+\frac{2}{\varepsilon_{0}} X^{T} P P \Delta+\varepsilon_{0} \delta^{2} \Delta_{2}^{T} \Delta_{2} \\
& +\varepsilon_{0} k_{5}^{2}-2\left[\left\lfloor\Delta_{1}\right\rfloor^{\alpha}\right]^{T} P_{1}\left(k_{1}\left\lfloor\Delta_{1}\right\rfloor^{\gamma_{1}}+k_{2} \Delta_{1}+k_{3}\left\lfloor\Delta_{1}\right\rfloor^{\gamma_{2}}\right) \\
& +2 p_{1}\left[\left\lfloor\Delta_{1}\right\rfloor^{\alpha}\right]^{T} P_{1}\left(\operatorname{col}\left(\left(a_{1} N_{1 i}\left(\xi_{1 i}\right)-1\right)\right)\right) \\
& +2 p_{2} \Delta_{2}^{T} P_{2} \operatorname{col}\left(\left(a_{2} N_{2 i}\left(\xi_{2 i}\right)-1\right)\right)
\end{aligned}
$$

According to (10), Eq. 20 can be further simplified as

$$
\begin{aligned}
\dot{V}_{1} & \leq X^{T}\left(P A_{0}+A_{0}^{T} P+\frac{2}{\varepsilon_{0}} P P+\Psi\right) \Delta+\varepsilon_{0} k_{5}^{2} \\
& +2 p_{1} / k_{4} \dot{\xi}_{1}^{T} P_{1}\left(\operatorname{col}\left(\left(a_{1} N_{1 i}\left(\xi_{1 i}\right)-1\right)\right)\right) \\
& +2 p_{2} / k_{6} \dot{\xi}_{2}^{T} P_{2} \operatorname{col}\left(\left(a_{2} N_{2 i}\left(\xi_{2 i}\right)-1\right)\right) \\
& \leq X^{T} Q \Delta+\varepsilon_{0} k_{5}^{2}+2 p_{1} / k_{4} \sum_{i=1}^{6} \dot{\xi}_{1 i} P_{1 i}\left(a_{1} N_{1 i}\left(\xi_{1 i}\right)-1\right) \\
& +2 p_{2} / k_{6} \sum_{i=1}^{6} \dot{\xi}_{2 i} P_{2 i}\left(a_{2} N_{2 i}\left(\xi_{2 i}\right)-1\right) .
\end{aligned}
$$

From the expression of $Q$, one has that $Q$ is a symmetric matrix. And if the matrix $Q$ is negative-definite matrix, i.e., the minimum eigenvalue of the matrix $Q$ is negative, it follows that $X^{T} Q \Delta \leq 0$. Then multiplying both sides of Eq. 21 by $\exp \left(c_{a} t\right)$ gives

$$
\begin{aligned}
\frac{d}{d t}\left(V_{1} \exp \left(c_{a} t\right)\right) & \leq \varepsilon_{0} k_{5}^{2} \exp \left(c_{a} t\right)-c_{a} V_{1} \exp \left(c_{a} t\right) \\
& +2 p_{1} / k_{4} \sum_{i=1}^{6} \dot{\xi}_{1 i} \Omega_{1 i} \exp \left(c_{a} t\right) \\
& +2 p_{2} / k_{6} \sum_{i=1}^{6} \dot{\xi}_{2 i} \Omega_{2 i} \exp \left(c_{a} t\right) \\
& \leq \varepsilon_{0} k_{5}^{2} \exp \left(c_{a} t\right) \\
& +2 p_{1} / k_{4} \sum_{i=1}^{6} \dot{\xi}_{1 i} \Omega_{1 i} \exp \left(c_{a} t\right) \\
& +2 p_{2} / k_{6} \sum_{i=1}^{6} \dot{\xi}_{2 i} \Omega_{2 i} \exp \left(c_{a} t\right)
\end{aligned}
$$

where $\Omega_{1 i}=P_{1 i}\left(a_{1} N_{1 i}\left(\xi_{1 i}\right)-1\right)$ and $\Omega_{2 i}=P_{2 i}\left(a_{2} N_{2 i}\left(\xi_{2 i}\right)-1\right)$

Integrating Eq. 22 on $[0, t]$ yields 


$$
\begin{aligned}
V_{1}(t) & \leq \frac{\varepsilon_{0} k_{5}^{2}}{c_{a}}+\left(V_{1}(0)-\frac{\varepsilon_{0} k_{5}^{2}}{c_{a}}\right) \exp \left(-c_{a} t\right) \\
& +2 p_{1} / k_{4} \exp \left(-c_{a} t\right) \sum_{i=1}^{6} \int_{0}^{t} \dot{\xi}_{1 i} \Omega_{1 i} \exp \left(c_{a} \tau\right) d \tau \\
& +2 p_{2} / k_{6} \exp \left(-c_{a} t\right) \sum_{i=1}^{6} \int_{0}^{t} \dot{\xi}_{2 i} \Omega_{2 i} \exp \left(c_{a} \tau\right) d \tau \\
& \leq C+2 p_{1} / k_{4} \exp \left(-c_{a} t\right) \sum_{i=1}^{6} \int_{0}^{t} \dot{\xi}_{1 i} \Omega_{1 i} \exp \left(c_{a} \tau\right) d \tau \\
& +2 p_{2} / k_{6} \exp \left(-c_{a} t\right) \sum_{i=1}^{6} \int_{0}^{t} \dot{\xi}_{2 i} \Omega_{2 i} \exp \left(c_{a} \tau\right) d \tau
\end{aligned}
$$

According to Lemma1, it can be concluded that $V_{1}(t), \xi_{1}, \xi_{2}, \int_{0}^{t} \sum_{i=1}^{6} \dot{\xi}_{1 i} P_{1 i}\left(a_{1} N_{1 i}\left(\xi_{1 i}\right)-1\right) \exp \left(c_{a} \tau\right) d \tau$ and $\int_{0}^{t} \sum_{i=1}^{6} \dot{\xi}_{2 i} P_{2 i}\left(a_{2} N_{2 i}\left(\xi_{2 i}\right)-1\right) \exp \left(c_{a} \tau\right) d \tau$ are bounded on $\left[0, t_{f}\right]$. And from 31,32 , it has that $t_{f}=\infty$. Therefore, it is verified that the estimation error $\Delta$ is uniformly ultimately bounded.

Next it needs to solve the inequality 111. According to Schur complement lemma, (11) is equivalent to the following inequality. Referring to the existence of negative-definite matrix $Q$, after determining the values of $\varepsilon_{0}$ and $\delta$, we can select a proper $T_{1}$ to calculate the following inequality according to linear matrix inequality technique in priori 35 .

$$
\left[\begin{array}{cc}
P A+A^{T} P-S^{T} K^{T}-K S+\Psi & P \\
P & -\frac{\varepsilon_{0}}{2} I_{12 \times 12}
\end{array}\right]<0
$$

where $S=\left[\begin{array}{ll}I_{6 \times 6} & 0_{6 \times 6}\end{array}\right], K=\left[\begin{array}{ll}P_{1} L_{1} & P_{2} L_{2}\end{array}\right]^{T}$.

It should be noted that $T_{1}$ should be select to guarantee that the solution of the above inequality exists. After obtaining the solution $P$ and $K$, the values of $L_{1}$ and $L_{2}$ are also found, i.e., $L=\left[L_{1}, L_{2}\right]=P^{-1} K$.

\subsection{Region tracking control design}

After using the Nussbaum state observer to estimate the unmeasured state $x_{2}$, this subsection will use these estimated states to design region tracking control law.

At first, define the tracking error $e_{1}=x_{1}-x_{1 d}$, where $x_{1 d}$ is the desired trajectory. As discussed in Section 1. the tracking error would converge to zero, if directly adopting prescribed performance control scheme, which is not the purpose of the region tracking. Therefore, a special transformation about the tracking error is designed to achieve region tracking, shown below.

$H_{n}(s, d)= \begin{cases}\frac{(|s|-d)^{n}}{n !}, & \text { if }|s| \geq d \\ 0, & \text { otherwise. }\end{cases}$

where $s$ is a variable, $d$ is a positive scale, $n$ is a positive integer.

And the partial derivative of $H_{n}(s, d)$ with respect to $s$ is given by $\frac{\partial}{\partial s} H_{n}(s, d)=H_{n-1}(s, d) \operatorname{sign}(s)$, for $n \geq 2$.

Consider the following Lyapunov function:

$V_{2}=\frac{1}{2} \sum_{i=1}^{6} \ln \frac{\rho_{a i}}{\rho_{a i}-z_{1 i}}$

where $z_{1 i}=H_{3}\left(e_{1 i}, \varepsilon_{1 i}\right), z_{1 i}, e_{1 i}, \rho_{a i}$ and $\varepsilon_{1 i}$ are the $i$ th entry of $z_{1}, e_{1}, \rho_{a}$ and $\varepsilon_{1}$, respectively. $\varepsilon_{1}$ is a positive vector. $\rho_{a i}$ is a function about the prescribed performance, describing the requirements of the transientstate and steady-state performances. Here it is set that $\rho_{a i}=\left(\left(\bar{\rho}_{i}-\underline{\rho}_{i}\right) \exp \left(-\mu_{\rho} t\right)+\underline{\rho}_{i}-\varepsilon_{1 i}\right)^{3} / 6$, where $\bar{\rho}, \underline{\rho}$ are positive vectors (their entries are $\bar{\rho}_{i}$ and $\rho_{i}$ ) and $\mu_{\rho}$ is positive constant with $\bar{\rho}_{i}>\underline{\rho}_{i}>0$ and $\underline{\rho}_{i}>\varepsilon_{1 i}, i=$ $1,2, \ldots, 6$. The initial condition of $\rho_{a}$ should be chosen to guarantee that $\rho_{a i}(0)>z_{1 i}(0)$. From Eq. $(26)$, it is straightforward to verify that $V_{2}$ is positive and continuous in the set that $\rho_{a i}>z_{1 i}, i=1,2, \ldots, 6$. One also has that $V_{2} \rightarrow \infty$ as $z_{1 i} \rightarrow \rho_{a i}$. Let $V\left(z_{1}\right)=V_{2}\left(z_{1}\right)+U(\varsigma)$, where $\gamma_{a}(\|\varsigma\|) \leq U(\varsigma) \leq \gamma_{b}(\|\varsigma\|)$ with class $K_{\infty}$ functions $\gamma_{a}$ and $\gamma_{b}$. Based on [19, 36, 37], $\rho_{a i}(0)>z_{1 i}(0)$ and if the inequality $\dot{V}\left(z_{1}\right) \leq-c_{2} V\left(z_{1}\right)+\vartheta_{c}$ holds with the positive constants $c_{2}, \vartheta_{c}$, then $z_{1}(t)$ remains in the set that $\rho_{a i}>z_{1 i}(i=1,2, \ldots, 6) \forall t \in[0, \infty)$.

Then, define $e_{2}=\hat{x}_{2}-x_{2 c}$, and $x_{2 c}$ is from the following first-order filter:

$\theta \dot{x}_{2 c}+x_{2 c}=\alpha_{c}$,

where $\theta$ is a positive constant, less than one in general. $\alpha_{c}$ is the virtual control law. And $x_{2 c}$ follows $\alpha_{c}$ with a bound, i.e., $\left\|x_{2 c}-\alpha_{c}\right\| \leq y_{c}$ with a positive constant $y_{c}$. And the time-derivative of $z_{1}$ is expressed as the following equation, after substituting (4) and the estimation error of the Nussbaum state observer:

$$
\begin{aligned}
\dot{z}_{1} & =\operatorname{diag}\left(H_{2}\left(e_{1 i}, \varepsilon_{1 i}\right) \operatorname{sign}\left(e_{1 i}\right)\right) \dot{e}_{1} \\
& =\operatorname{diag}\left(H_{2}\left(e_{1 i}, \varepsilon_{1 i}\right) \operatorname{sign}\left(e_{1 i}\right)\right)\left(\dot{x}_{1}-\dot{x}_{1 d}\right) \\
& =\operatorname{diag}\left(H_{2}\left(e_{1 i}, \varepsilon_{1 i}\right) \operatorname{sign}\left(e_{1 i}\right)\right)\left(\hat{x}_{2}+T_{1} x_{1}+\Delta_{2}-\dot{x}_{1 d}\right),
\end{aligned}
$$


where the operation "diag" is to combine the elements into a $6 \times 6$ diagonal matrix, and $i=(1, \ldots, 6)$.

The virtual control law $\alpha_{c}$ considered in this paper has the following form:

$$
\begin{aligned}
\alpha_{c} & =-\frac{1}{3}\left(b_{1}-\frac{\dot{\rho}_{a i}}{\rho_{a i}}\right) \operatorname{col}\left(H_{1}\left(e_{1 i}, \varepsilon_{1 i}\right) \operatorname{sign}\left(e_{1 i}\right)\right) \\
& +\dot{x}_{1 d}-T_{1} x_{1} \\
& -\frac{1}{3} b_{2} \operatorname{col}\left(\frac{H_{1}\left(e_{1 i}, \varepsilon_{1 i}\right) \operatorname{sign}\left(e_{1 i}\right) z_{1 i}^{l_{1}}}{\left(\rho_{a i}-z_{1 i}\right)^{l_{1}}}\right) \\
& -\operatorname{col}\left(\frac{H_{2}\left(e_{1 i}, \varepsilon_{1 i}\right) \operatorname{sign}\left(e_{1 i}\right)}{\rho_{a i}-z_{1 i}}\right)
\end{aligned}
$$

where $b_{1}$ and $b_{2}$ are positive constants, $l_{1} \in(0,1)$.

Substituting (28) and 29 into the time derivative of $V_{2}$ yields

$$
\begin{aligned}
\dot{V}_{2} & =\sum_{i=1}^{6} \frac{-\dot{\rho}_{a i} z_{1 i}+\rho_{a i} \dot{z}_{1 i}}{\rho_{a i}\left(\rho_{a i}-z_{1 i}\right)} \\
& =\sum_{i=1}^{6} \frac{-\dot{\rho}_{a i} z_{1 i}}{\rho_{a i}\left(\rho_{a i}-z_{1 i}\right)}+\Upsilon_{\alpha}\left(\hat{x}_{2}+T_{1} x_{1}+\Delta_{2}-\dot{x}_{1 d}\right) \\
& =\sum_{i=1}^{6} \frac{-\dot{\rho}_{a i} z_{1 i}}{\rho_{a i}\left(\rho_{a i}-z_{1 i}\right)}+\Upsilon_{\alpha}\left(e_{2}+\alpha_{c}+T_{1} x_{1}-\dot{x}_{1 d}\right) \\
& +\Upsilon_{\alpha}\left(\Delta_{2}+x_{2 c}-\alpha_{c}\right) \\
& =-b_{1} \sum_{i=1}^{6} \frac{z_{1 i}}{\left(\rho_{a i}-z_{1 i}\right)}-b_{2} \sum_{i=1}^{6}\left(\frac{z_{1 i}}{\left(\rho_{a i}-z_{1 i}\right)}\right)^{1+l_{1}} \\
& +\sum_{i=1}^{6} \frac{z_{1 i} H_{2}\left(e_{1 i}, \varepsilon_{1 i}\right) \operatorname{sign}\left(e_{1 i}\right) e_{2 i}}{\left(\rho_{a i}-z_{1 i}\right)} \\
& -\sum_{i=1}^{6}\left(\frac{H_{2}\left(e_{1 i}, \varepsilon_{1 i}\right)}{\left(\rho_{a i}-z_{1 i}\right)}\right)^{2}+\Upsilon_{\alpha}\left(\Delta_{2}+x_{2 c}-\alpha_{c}\right),
\end{aligned}
$$

where $\Upsilon_{\alpha}=\operatorname{row}\left(\frac{1}{\rho_{a i}-z_{1 i}}\right) \operatorname{diag}\left(H_{2}\left(e_{1 i}, \varepsilon_{1 i}\right) \operatorname{sign}\left(e_{1 i}\right)\right)$, the operation "row" is to combine entries into a row vector, for example, $\operatorname{row}\left(Y_{i}\right)=\left[Y_{1}, Y_{2}, \ldots, Y_{N}\right]$.

By employing the Young's inequality [34], the following inequalities can be obtained

$$
\begin{aligned}
\Upsilon_{\alpha} \Delta_{2} & \leq \frac{1}{2} \sum_{i=1}^{6}\left(\frac{H_{2}\left(e_{1 i}, \varepsilon_{1 i}\right)}{\left(\rho_{a i}-z_{1 i}\right)}\right)^{2}+\frac{1}{2} \Delta_{2}^{T} \Delta_{2} \\
\Upsilon_{\alpha}\left(x_{2 c}-\alpha_{c}\right) & \leq \frac{1}{2} \sum_{i=1}^{6}\left(\frac{H_{2}\left(e_{1 i}, \varepsilon_{1 i}\right)}{\left(\rho_{a i}-z_{1 i}\right)}\right)^{2}+\frac{1}{2} y_{c}^{2} .
\end{aligned}
$$

Consequently, substituting the above two inequalities into 30 gives

$$
\begin{aligned}
\dot{V}_{2} & \leq-b_{1} \sum_{i=1}^{6} \frac{z_{1 i}}{\left(\rho_{a i}-z_{1 i}\right)}-b_{2} \sum_{i=1}^{6}\left(\frac{z_{1 i}}{\left(\rho_{a i}-z_{1 i}\right)}\right)^{1+l_{1}} \\
& +\sum_{i=1}^{6} \frac{z_{1 i} H_{2}\left(e_{1 i}, \varepsilon_{1 i}\right) \operatorname{sign}\left(e_{1 i}\right) e_{2 i}}{\left(\rho_{a i}-z_{1 i}\right)}+\frac{1}{2} \Delta_{2}^{T} \Delta_{2}+\frac{1}{2} y_{c}^{2} .
\end{aligned}
$$

Next, differentiating $e_{2}$ with respect to time, and then substituting the estimation error of the Nussbaum state observer and (4) obtains

$$
\begin{aligned}
\dot{e}_{2} & =\dot{\hat{x}}_{2}-\dot{x}_{2 c}=\dot{\hat{x}}_{2}-\frac{\left(x_{2 c}-\alpha_{c}\right)}{\theta} \\
& =\dot{x}_{2}-\frac{\left(x_{2 c}-\alpha_{c}\right)}{\theta}-\dot{\Delta}_{2} \\
& =-T_{1}^{2} x_{1}-T_{1} x_{2}+\bar{F}\left(x_{1}, x_{2}\right)+E\left(x_{1}\right) u \\
& -\frac{\left(x_{2 c}-\alpha_{c}\right)}{\theta}-\dot{\Delta}_{2} .
\end{aligned}
$$

Neural network is always used to approximate the unknown function in control designs. Here, a RBF neural network is used to approximate the unknown function $\bar{F}\left(x_{1}, x_{2}\right)$. Of course, another type of neural networks can be also applied. Considering that the ocean current and the velocity of the vehicle are bounded, it is reasonable that the unknown function $\bar{F}\left(x_{1}, x_{2}\right)$ can be approximated on a compact set. Define $\Gamma$ be the compact set. Due to the approximation property of RBF neural network, there exits an ideal weighting matrix $W^{*} \in \mathbb{R}^{6 \times m}$, where $m$ denotes the number of neurons in the hidden layer. $W^{*}$ satisfies $\left\|W^{*}\right\| \leq \bar{W}$ with $\bar{W}>0$, such that 38,39

$\bar{F}\left(x_{1}, x_{2}\right)=W^{*} \Phi\left(\hat{x}_{1}, \hat{x}_{2}\right)+\varepsilon_{f}$,

where $\Phi\left(\hat{x}_{1}, \hat{x}_{2}\right)=\left[\Phi_{1}\left(\hat{x}_{1}, \hat{x}_{2}\right), \Phi_{2}\left(\hat{x}_{1}, \hat{x}_{2}\right), \ldots, \Phi_{m}\left(\hat{x}_{1}, \hat{x}_{2}\right)\right]$, $\Phi_{i}\left(\hat{x}_{1}, \hat{x}_{2}\right)=\exp \left(-\left\|\left(\hat{x}_{2}+T_{1} \hat{x}_{1}\right)-\mu_{i}\right\|^{2} / \sigma_{i}^{2}\right), \sigma_{i}$ is the width of the $i$ th neuron, while $\mu_{i}$ is the center vector of the $i$ th neuron. The input of neural network is the estimated quantity of the vehicle's velocity, i.e., $\left(\hat{x}_{2}+T_{1} \hat{x}_{1}\right)$, since $\bar{F}\left(x_{1}, x_{2}\right)$ is mainly affected by the vehicle's velocity from our previous experiences. And $\varepsilon_{f}$ is the approximation error of neural network, affected by the number of neurons and network structure. It is always assumed that the approximation error is bounded with a positive bound $\Xi$, i.e., $\left\|\varepsilon_{f}\right\| \leq \Xi$.

The output of the RBF neural network is given by

$\bar{F}^{e}\left(\hat{x}_{1}, \hat{x}_{2}\right)=\hat{W} \Phi\left(\hat{x}_{1}, \hat{x}_{2}\right)$ 
where $\hat{W}$ is the estimated weighting matrix of $W^{*}$, and $\tilde{W}=W^{*}-\bar{W}$.

Therefore, the control law $u$ is designed with the following form:

$$
\begin{aligned}
u & =u_{0}+u_{1} \\
u_{0} & =E\left(x_{1}\right)^{+}\left(T_{1}^{2} x_{1}+T_{1} \hat{x}_{2}+\frac{\left(x_{2 c}-\alpha_{c}\right)}{\theta}-\hat{W} \Phi\left(\hat{x}_{1}, \hat{x}_{2}\right)\right) \\
& -E\left(x_{1}\right)^{+} \operatorname{col}\left(\frac{H_{2}\left(e_{1 i}, \varepsilon_{1 i}\right) \operatorname{sign}\left(e_{1 i}\right)}{\rho_{a i}-z_{1 i}}\right) \\
u_{1} & =E\left(x_{1}\right)^{+}\left(-b_{3} e_{2}-\hat{\Xi} \operatorname{col}\left(\tanh \left(\frac{e_{2 i}}{\rho_{b}}\right)\right)\right),
\end{aligned}
$$

where $E\left(x_{1}\right)^{+}$is the pseudo-inverse matrix of $E\left(x_{1}\right) . b_{3}$ and $\rho_{b}$ are the positive constants. $\hat{\Xi}$ is the estimated quantity of $\Xi$, given by

$$
\begin{aligned}
\dot{\hat{W}} & =\Gamma_{1} e_{2}^{T} \Phi\left(\hat{x}_{1}, \hat{x}_{2}\right)-\beta_{1} \hat{W} \\
\dot{\hat{\Xi}} & =\Gamma_{2} e_{2}^{T} \operatorname{col}\left(\tanh \left(\frac{e_{2 i}}{\rho_{b}}\right)\right)-\beta_{2} \hat{\Xi}
\end{aligned}
$$

where $\Gamma_{1}, \Gamma_{2}, \beta_{1}$ and $\beta_{2}$ are positive constants.

Then consider the following Lyapunov function

$V_{3}=\frac{1}{2} e_{2}^{T} e_{2}+\frac{1}{2 \Gamma_{1}} \operatorname{tr}\left(\tilde{W}^{T} \tilde{W}\right)+\frac{1}{2 \Gamma_{2}} \tilde{\Xi}^{2}$,

where $\tilde{\Xi}=\Xi-\hat{\Xi} \cdot \operatorname{tr}\left(\tilde{W}^{T} \tilde{W}\right)$ means to take the trace of the matrix $\left(\tilde{W}^{T} \tilde{W}\right)$. And differentiating both sides of 39 with respect to time yields

$$
\begin{aligned}
& \dot{V}_{3}=e_{2}^{T}\left(-T_{1}^{2} x_{1}-T_{1} x_{2}+\bar{F}\left(x_{1}, x_{2}\right)+E\left(x_{1}\right) u\right) \\
& -e_{2}^{T}\left(\frac{\left(x_{2 c}-\alpha_{c}\right)}{\theta}+\dot{\Delta}_{2}\right)-\frac{1}{\Gamma_{1}} \operatorname{tr}\left(\tilde{W}^{T} \dot{ }{ }^{\dot{1}} W\right)-\frac{1}{\Gamma_{2}} \tilde{\Xi} \dot{\Xi} \Xi \\
& =e_{2}^{T}\left(-\operatorname{col}\left(\frac{H_{2}\left(e_{1 i}, \varepsilon_{1 i}\right) \operatorname{sign}\left(e_{1 i}\right)}{\rho_{a i}-z_{1 i}}\right)+\tilde{W} \Phi\left(\hat{x}_{1}, \hat{x}_{2}\right)\right) \\
& +e_{2}^{T}\left(\varepsilon_{f}-\dot{\Delta}_{2}-b_{3} e_{2}-\hat{\Xi} \operatorname{col}\left(\tanh \left(\frac{e_{2 i}}{\rho_{b}}\right)\right)\right) \\
& -e_{2}^{T} \tilde{W} \Phi\left(\hat{x}_{1}, \hat{x}_{2}\right)+\frac{\beta_{1}}{\Gamma_{1}} \operatorname{tr}\left(\tilde{W}^{T} \hat{W}\right) \\
& -\tilde{\Xi} e_{2}^{T} \operatorname{col}\left(\tanh \left(\frac{e_{2 i}}{\rho_{b}}\right)\right)-\frac{\beta_{2}}{\Gamma_{2}} \hat{\Xi} \\
& =-e_{2}^{T}\left(\operatorname{col}\left(\frac{H_{2}\left(e_{1 i}, \varepsilon_{1 i}\right) \operatorname{sign}\left(e_{1 i}\right)}{\rho_{a i}-z_{1 i}}\right)\right) \\
& -e_{2}^{T}\left(b_{3} e_{2}+\hat{\Xi} \operatorname{col}\left(\tanh \left(\frac{e_{2 i}}{\rho_{b}}\right)\right)\right)+e_{2}^{T}\left(\varepsilon_{f}-\dot{\Delta}_{2}\right) \\
& +\frac{\beta_{1}}{\Gamma_{1}} \operatorname{tr}\left(\tilde{W}^{T} \hat{W}\right)-\tilde{\Xi} e_{2}^{T} \operatorname{col}\left(\tanh \left(\frac{e_{2 i}}{\rho_{b}}\right)\right)-\frac{\beta_{2}}{\Gamma_{2}} \hat{\Xi} .
\end{aligned}
$$

By employing the Young's inequality, the following inequalities can be obtained

$$
\begin{aligned}
e_{2}^{T} \varepsilon_{f}-\hat{\Xi} e_{2}^{T} \operatorname{col}\left(\tanh \left(\frac{e_{2 i}}{\rho_{b}}\right)\right) & \leq \tilde{\Xi} e_{2}^{T} \operatorname{col}\left(\tanh \left(\frac{e_{2 i}}{\rho_{b}}\right)\right) \\
& +6 \varsigma \rho_{b} \Xi \\
e_{2}^{T} \dot{\Delta}_{2} & \leq \frac{1}{2} e_{2}^{T} e_{2}+\frac{1}{2}\left\|\dot{\Delta}_{2}\right\|^{2} \\
\operatorname{tr}\left(\tilde{W}^{T} \hat{W}\right) & \leq \frac{1}{2} \operatorname{tr}\left(W^{* T} W^{*}\right) \\
& -\frac{1}{2} \operatorname{tr}\left(\tilde{W}^{T} \tilde{W}\right) \\
\tilde{\Xi} \hat{\Xi} & \leq \frac{1}{2} \Xi^{2}-\frac{1}{2} \tilde{\Xi}^{2}
\end{aligned}
$$

Then, substituting the above four inequalities into (40) gives

$$
\begin{aligned}
\dot{V}_{3} & \leq-e_{2}^{T}\left(\operatorname{col}\left(\frac{H_{2}\left(e_{1 i}, \varepsilon_{1 i}\right) \operatorname{sign}\left(e_{1 i}\right)}{\rho_{a i}-z_{1 i}}\right)+b_{3} e_{2}\right) \\
& -\frac{\beta_{1}}{2 \Gamma_{1}} \operatorname{tr}\left(\tilde{W}^{T} \tilde{W}\right)-\frac{\beta_{2}}{2 \Gamma_{2}} \tilde{\Xi}^{2}+\frac{\beta_{1}}{2 \Gamma_{1}} \operatorname{tr}\left(W^{* T} W^{*}\right) \\
& +\frac{\beta_{2}}{2 \Gamma_{2}} \Xi^{2}+6 \varsigma \rho_{b} \Xi+\frac{1}{2}\left\|\dot{\Delta}_{2}\right\|^{2} .
\end{aligned}
$$

Now, the main result of this paper is given as below.

Theorem 1 Consider an underwater vehicle without velocity measurement (1). Given the performance function $\rho_{a i}, i=1,2, \ldots, 6$ with the constraint that $\rho_{a i}(0)>$ $z_{1 i}(0)$. If the state observer is designed as the form in (7) and (9), and the region tracking control law is presented as (37) together with the adaptive laws as (38), then the following properties always hold.

(1) all the signals in the closed-loop system, including $z_{1}, e_{2}, \tilde{W}$ and $\tilde{\Xi}$, are uniformly ultimately bounded;

(2) the tracking error $e_{1 i}$ is always kept within the prescribed boundaries $\left(\bar{\rho}_{i}-\underline{\rho}_{i}\right) \exp \left(-\mu_{\rho} t\right)+\underline{\rho}_{i}$ for $i=$ $1,2, \ldots, 6$, i.e., the region tracking control is achieved.

Proof To demonstrate the convergence of the signals in the closed-loop systems, the following Lyapunov function is considered

$V=V_{2}+V_{3}$

Then differentiating both sides of 46 , and substituting 33 and 45 yields 


$$
\begin{aligned}
\dot{V} & \leq-b_{1} \sum_{i=1}^{6} \frac{z_{1 i}}{\left(\rho_{a i}-z_{1 i}\right)}-b_{2} \sum_{i=1}^{6}\left(\frac{z_{1 i}}{\left(\rho_{a i}-z_{1 i}\right)}\right)^{1+l_{1}} \\
& -b_{3} e_{2}^{T} e_{2}-\frac{\beta_{1}}{2 \Gamma_{1}} \operatorname{tr}\left(\tilde{W}^{T} \tilde{W}\right)-\frac{\beta_{2}}{2 \Gamma_{2}} \tilde{\Xi}^{2} \\
& +\frac{1}{2} \Delta_{2}^{T} \Delta_{2}+\frac{1}{2} y_{c}^{2}+\frac{\beta_{1}}{2 \Gamma_{1}} \operatorname{tr}\left(W^{* T} W^{*}\right)+\frac{\beta_{2}}{2 \Gamma_{2}} \Xi^{2} \\
& +6 \varsigma \rho_{b} \Xi+\frac{1}{2}\left\|\dot{\Delta}_{2}\right\|^{2} .
\end{aligned}
$$

According to [40], for all $z_{1 i}$ in the set $z_{1 i}<\rho_{a}$, the following inequality holds

$$
\sum_{i=1}^{6} \ln \frac{\rho_{a i}}{\rho_{a i}-z_{1 i}} \leq \sum_{i=1}^{6} \frac{z_{1 i}}{\left(\rho_{a i}-z_{1 i}\right)} .
$$

Consequently, 47 can be further rewritten as

$$
\begin{aligned}
\dot{V} & \leq-b_{1} V_{2}-b_{2} V_{2}^{1+l_{1}}-\lambda_{1} V_{3}+\bar{C}_{0} \\
& \leq-\lambda_{2} V+\bar{C}_{0},
\end{aligned}
$$

where $\lambda_{1}=\min \left(2 b_{3}, \beta_{1}, \beta_{2}\right)>0, \lambda_{2}=\min \left(\lambda_{1}, b_{1}\right)>$ 0. $\bar{C}_{0}=\frac{1}{2} \Delta_{2}^{T} \Delta_{2}+\frac{1}{2} y_{c}^{2}+\frac{\beta_{1}}{2 \Gamma_{1}} \operatorname{tr}\left(W^{* T} W^{*}\right)+\frac{\beta_{2}}{2 \Gamma_{2}} \Xi^{2}+$ $6 \varsigma \rho_{b} \Xi+\frac{1}{2}\left\|\dot{\Delta}_{2}\right\|^{2}$. Since $\Delta_{2}, \dot{\Delta}_{2}, y_{c}, W^{*}, \Xi$ are bounded, $\bar{C}_{0}$ is also bounded.

Integrating Eq. 499) over $[0, t]$, one has

$$
\begin{aligned}
0 \leq V(t) & \leq \frac{\bar{C}_{0}}{\lambda_{2}}+\left(V(0)-\frac{\bar{C}_{0}}{\lambda_{2}}\right) \exp \left(-\lambda_{2} t\right) \\
& \leq V(0) \exp \left(-\lambda_{2} t\right)+\frac{\bar{C}_{0}}{\lambda_{2}}
\end{aligned}
$$

Due to the constraint that $\rho_{a i}(0)>z_{1 i}(0)$, it is obviously that $V(0)>0$. Therefore one has that $V(t)$ is uniformly ultimately bounded according to 41 . Then in light of (46), all the signals of the closed-loop system, including $z_{1}, e_{2}, \tilde{W}$ and $\tilde{\Xi}$, are uniformly ultimately bounded. The proof of Result 1 is completed. Furthermore, one has that $z_{1 i} \leq \rho_{a i}, i=1,2, \ldots, 6$ for any $t>0$ when the initial condition is satisfied, according to the research in 19, 36, 37.

Then, according to the definition of the variable $z_{1}$ and $\rho_{a}$, for the case of $\left|e_{1 i}\right| \geq \varepsilon_{1 i}$, one has the following inequality:

$$
\begin{aligned}
0 & \leq \frac{\left(\left|e_{1 i}\right|-\varepsilon_{1 i}\right)^{3}}{3 !}<\rho_{a i} \\
\varepsilon_{1 i} & \leq\left|e_{1 i}\right|<\left(\bar{\rho}_{i}-\underline{\rho}_{i}\right) \exp \left(-\mu_{\rho} t\right)+\underline{\rho}_{i} .
\end{aligned}
$$

From Eq.51], it can be obtained that the tracking error $e_{1 i}$ is always kept within the prescribed boundaries defined below Eq. 26. The proof of Result 2 is completed.

\section{Simulation studies}

This section presents a series of simulation results to demonstrate the effectiveness and feasibility of the proposed observer based region tracking control scheme for underwater vehicles without velocity measurement. And two control schemes are used to conduct comparative studies, including a traditional observer-based backstepping control scheme and a traditional region tracking control with high-gain observer.

As usual, a typical representation of fully-actuated underwater vehicles is considered. The vehicle's dynamic parameters and thruster distribution are presented in [42. The initial condition of the vehicle is set as $\eta(0)=$ $[1,1,-1,2 \pi / 9,2 \pi / 9,2 \pi / 9]^{T}$, and the velocity $\dot{\eta}(0)=$ $[0.04,0.04,0.04,0.02,0.02,0.02]^{T}$.

To reflect the effectiveness of the proposed observer based region tracking control scheme to reject ocean current disturbances, the following equation is used to generate ocean current $\dot{\eta}_{c}$ 21,30:

$\frac{d}{d t}\left\|\dot{\eta}_{c}\right\|+\mu_{c}\left\|\dot{\eta}_{c}\right\|=\omega_{c}$

where $\left\|\dot{\eta}_{c}\right\|$ denotes the magnitude of ocean current $\dot{\eta}_{c} ; \mu_{c}$ is a fixed constant, set as $3 ; \omega_{c}$ is a Gaussian noise with mean 1.5 and variance 1 . The orientation of ocean current is simulated by the sum of Gaussian noise with mean 0 and variance 50 . According to the model, the mean value of ocean current used in simulations is $0.50 \mathrm{~m} / \mathrm{s}$ while its standard variance is 0.056 .

This paper uses a RBF neural network to approximate the unknown function $\bar{F}\left(x_{1}, x_{2}\right)$. Only one hidden layer is considered, and the number of the neurons in the hidden layer is 54. $\sigma_{i}=0.1$ and the centers of radial basis functions are distributed evenly within the range of $[-0.5,0.5] \times[-0.5,0.5] \times[-0.5,0.5] \times[-0,0] \times[-0,0] \times$ $[-0.2,0.2]$. Also, the initial entries of the weighting matrix $\hat{W}$ are zero.

Solving (24) using LMI technique, then the following parameters are determined. $T_{1}=2.5 I_{6 \times 6}, P_{1}=$ $0.0641 I_{6 \times 6}, P_{2}=0.0641 I_{6 \times 6}, L_{1}=4.365 I_{6 \times 6}, L_{2}=$ $-5.25 I_{6 \times 6}$.

According to the definitions of variables $\alpha, \gamma_{1}$ and $\gamma_{2}$, the values of these variables are given as $\alpha=0.5$, $\gamma_{1}=1 / 3, \gamma_{2}=1.5$. And two types Nussbaum-type 
functions are used in the observer,including $N_{1 i}\left(\xi_{1 i}\right)=$ $\xi_{1 i}^{2} \cos \left(\xi_{1 i}\right), N_{2 i}\left(\xi_{2 i}\right)=\xi_{2 i}^{2} \cos \left(\xi_{2 i}\right)$.

In order not to introduce serious fluctuation in the estimated velocity, $k_{1}, k_{3}, k_{5}, a_{1}, a_{2}$ should be set relatively small values.Here, these variables are set as $k_{1}=$ $1, k_{3}=1, k_{5}=0.5, a_{1}=0.1, a_{2}=0.1$. After determining these values of the above variables, the other parameters used in the observer are selected by trial and error. Here, $k_{0}=10, k_{2}=10, k_{4}=10, k_{6}=10$, $p_{1}=0.1+\tanh (t / 30), p_{2}=0.1+\tanh (t / 30)$.

The prescribed performance function is selected according to the given mission. For example, here the following function is chosen. $\epsilon_{1 i}=0.1,(i=1,2, \ldots, 6)$ and $\rho_{a}=((2-0.3) \exp (-0.1 t)+(0.3-0.1))^{3} / 6$. Of course, these above parameters can be changed as the requirement of the given mission. Finally, the control parameters are selected as below.

$b_{1}=0.5, b_{2}=0.5, \theta=0.1, l_{1}=1 / 3, b_{3}=2, \rho_{b}=2$. $\Gamma_{1}=0.5, \beta_{1}=0.6, \Gamma_{2}=0.1, \beta_{2}=0.05$.

In simulations, the vehicle is commanded to track the following desired trajectory:

$\eta_{d}=\left[x_{d}, y_{d}, z_{d}, 0,0,0\right]^{T}$,

where $x_{d}=4(\sin (0.05 t)+\sin (0.15 t)), z_{d}=-2, y_{d}=$ $4(\cos (0.05 t)-\cos (0.15 t))$.

4.1 Simulation verification for the case without measurement noise

In this subsection, the case without measurement noise is considered to verify the effectiveness of the proposed observer-based region tracking control strategy, where two comparative control algorithms are considered. Specifically, a traditional observer-based backstepping control algorithm is used to demonstrate the advantages of the region tracking control strategy, while a traditional region tracking control scheme based on high-gain observer is applied to verify the superior of the proposed region tracking control strategy.

The form of the traditional observer-based backstepping controller is shown as

$$
\begin{aligned}
\alpha_{c} & =-T_{1} x_{1}+\dot{x}_{1 d}-b_{1} e_{1}-b_{2}\left\lfloor e_{1}\right\rfloor^{\gamma_{1}} \\
\dot{x}_{2 c} & =\left(\alpha_{c}-x_{2 c}\right) / \theta \\
e_{2} & =\hat{x}_{2}-x_{2 c} \\
u & =u_{0}+u_{1} \\
u_{0} & =E\left(x_{1}\right)^{+}\left(T_{1}^{2} x_{1}+T_{1} \hat{x}_{2}+\frac{\left(x_{2 c}-\alpha_{c}\right)}{\theta}\right) \\
& -E\left(x_{1}\right)^{+} \hat{W} \Phi\left(\hat{x}_{1}, \hat{x}_{2}\right),
\end{aligned}
$$

$$
u_{1}=E\left(x_{1}\right)^{+}\left(-b_{3} e_{2} b_{4}\left\lfloor e_{2}\right\rfloor^{\gamma_{2}}-\hat{\Xi} \operatorname{col}\left(\tanh \left(\frac{e_{2 i}}{\rho_{b}}\right)\right)\right) \text {, }
$$

where $T_{1}=2.5 I_{6 \times 6}, b_{1}=3, b_{2}=1, b_{3}=2, b_{4}=$ $2, \gamma_{1}=\gamma_{2}=1 / 3, \theta=0.2, \rho_{b}=2$. In addition, the weighting matrix $\hat{W}$ and the parameter $\hat{\Xi}$ are still from (38), and the estimated quantities $\hat{x}_{1}$ and $\hat{x}_{2}$ are from the observer (7).

The traditional region tracking control scheme is from 13, 14. Since the control law is designed based on the full states of an underwater vehicle, high-gain observer is added to estimate the unmeasured velocity. Hence, the form of the traditional region tracking control scheme based on high-gain observer is given as follows:

$$
\begin{aligned}
F p\left(e_{1}\right) & =\sum_{i=1}^{6} \frac{\kappa_{i}}{2}\left(\max \left(0, f p_{i}\left(e_{1 i}\right)\right)\right)^{2} \\
v_{s} & =J^{-1}(\eta)\left(\dot{\eta}_{d}-\alpha_{v}\left(\frac{\partial F p\left(e_{1}\right)}{\partial e_{1}}\right)^{T}\right) \\
u & =B^{+}\left(-\hat{W} \Phi(0, \hat{v})-M \dot{v}_{s}-K_{d} \tilde{v}-k_{s} \operatorname{sign}(\tilde{v})\right) \\
& -B^{+} J^{T}(\eta) K_{p}\left(\frac{\partial F p\left(e_{1}\right)}{\partial e_{1}}\right)^{T},
\end{aligned}
$$

where $f p\left(e_{1}\right)=\left[f p_{1}\left(e_{11}\right), f p_{2}\left(e_{12}\right), \ldots f p_{6}\left(e_{16}\right)\right]^{T}$, and $f p_{i}\left(e_{1 i}\right)=0.5\left(e_{1 i}^{2}-r_{i}^{2}\right)$ with the individual regional bound vector $r, i=(1,2, \ldots, 6) . \hat{W}$ comes from Eq. 38. $\kappa$ and $\alpha_{v}$ are positive constants. $\tilde{v}=\hat{v}-v_{s}, \hat{v}$ is the estimated velocity of the vehicle with respect to the bodyfixed frame $\hat{v}=J^{-1}(\eta) \hat{x}_{2}$ from the following high-gain observer:

$$
\begin{aligned}
\dot{\hat{x}}_{1} & =T_{1} \hat{x}_{1}+\hat{x}_{2}+L_{1} \Delta_{1} \\
\dot{\hat{x}}_{2} & =-T_{1}^{2} \hat{x}_{1}-T_{1} \hat{x}_{2}+M_{\eta}^{-1}(\eta) \hat{W} \Phi(0, \hat{v}) \\
& +E\left(x_{1}\right) u+L_{2} \Delta_{1},
\end{aligned}
$$

where $T_{1}=0, L_{1}=100, L_{2}=2000$.

Under the action of the proposed observer-based region tracking control strategy, the desired path and the real path are shown in Fig. 2, and the tracking results of the vehicle are shown in Fig. 3, where the dash line denotes the prescribed boundaries. From Fig. 2 it is obvious that the vehicle did not accurately follow the desired path under the proposed observer-based region tracking control strategy. That is because that the high tracking precision is not the priority of the region tracking control, as long as the tracking errors are always kept within the prescribed boundaries as shown 
in Fig. 3(a). The corresponding control inputs of each thrusters are presented in Fig. 3(b). Fig. 4 is the results of the conventional backstepping control scheme based on the Nussbaum state observer. It is shown from Fig. 3(a) and Fig. 4(a) that the tracking precision of the conventional backstepping control scheme is better than the proposed control scheme, while the chattering phenomenon are much more serious. From Fig. 3 and Fig. 4 the results demonstrate that the control inputs become relatively smooth by sacrificing a certain tracking precision and the tracking errors still satisfies the requirement of the prescribed boundaries under the action of the proposed observer based region tracking control strategy. Fig. 5 presents the tracking results, including tracking error and control input, based on the conventional region tracking control scheme based on high-gain observer, where the dash line denotes the prescribed boundaries. Compared with the proposed control strategy, the tracking errors cannot be maintain within the prescribed region under the action of the conventional region tracking control scheme, although the control inputs are relatively smooth in comparison with the conventional backstepping control scheme.

It is be highlighted that the tracking accuracy is not the first priority in the region tracking control, as long as the tracking error satisfies the prescribed requirements/boundaries. Hence, the indexes about control accuracy in the normal tracking control are not used to explain the advantages of the proposed region tracking control strategy. Contrarily, on the premise that the tracking error is always kept within the prescribed boundaries, we need to pay more attention on control inputs to demonstrate the performance of the region tracking control strategy. To be quantitatively evaluate the performances of the proposed observer-based region tracking control strategy, two indexes about control inputs are used, including energy consumption (i.e., the integral of the square of the control input) and chattering (i.e., the integral of the variance of control input), while whether the tracking error is always kept within the prescribed boundaries or not is considered the index about tracking precision, after all high precision is not the original purpose of the region tracking control concept.

Therefore, from Figs, 35 and Table 1] it can be concluded that under the action of the proposed region tracking control strategy, the tracking errors are within the prescribed boundaries all the time and meanwhile the control inputs are relatively smooth among these control strategies for underwater vehicles without measurement noise.

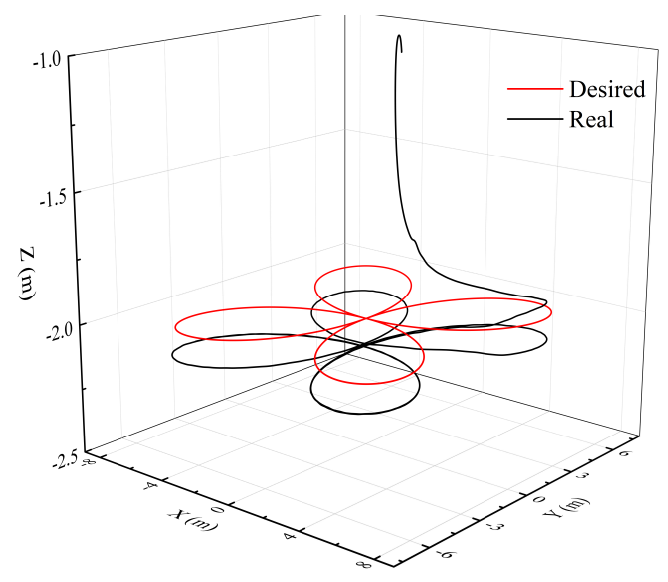

Fig. 2 Path considered in simulation

4.2 Simulation verification for the case with measurement noise

For an underwater vehicle, it is inevitable that the vehicle's state measured from sensors suffer from measurement noises. Hence, in this subsection, measurement noise is considered to verify the effectiveness of the proposed observer-based region tracking control strategy. The measurement noise is simulated as follows. Gaussian noise with mean 0 and variance 1 is filtered by a low-frequency filter and the result (change between 0.2 to 0.25 ) is added into the vehicle's position/attitude states.

Under the same measurement noise, the tracking results of these control strategies are, respectively, presented in Figs. 6,8, Among these control strategies, the the control inputs from the conventional backstepping control scheme are most easily to be affected by measurement noises, which is caused by the fact that the goal of this control strategy is to obtain high tracking precision. Compared Fig. 6 with Fig. 8, the tracking errors of the vehicle is always kept within the prescribed boundaries based on the proposed control strategy while the conventional region tracking control scheme is failed in terms of the region reachability. In order to clearly demonstrate the performances of these control strategies, similar to the case without measurement noises, the results about region reachability, evaluation about control inputs are tabulated in Table 2

From Figs. 6 8 and Table 2, it can be concluded that the proposed observer-based region tracking control strategy can guarantee the tracking errors to be always within the prescribed and its control inputs are relatively smooth among these control strategies even in presence of the measurement noises. 
Table 1 Comparative results of these control strategies for the case without measurement noise

\begin{tabular}{llll}
\hline Strategies & Region reachability & Energy consumption & Chattering \\
\hline Proposed & YES & $1.42 \times 10^{8}\left(N^{2}\right)$ & $5.02 \times 10^{3}(N)$ \\
Backstepping & YES & $1.85 \times 10^{8}\left(N^{2}\right)$ & $3.00 \times 10^{4}(N)$ \\
Conventional region & NO & $1.60 \times 10^{8}\left(N^{2}\right)$ & $1.28 \times 10^{4}(N)$ \\
\hline
\end{tabular}

Table 2 Comparative results of these control strategies for the case with measurement noise

\begin{tabular}{llll}
\hline Strategies & Region reachability & Energy consumption & Chattering \\
\hline Proposed & YES & $1.56 \times 10^{8}\left(N^{2}\right)$ & $6.30 \times 10^{3}(N)$ \\
Backstepping & YES & $2.15 \times 10^{8}\left(N^{2}\right)$ & $5.76 \times 10^{4}(N)$ \\
Conventional region & NO & $1.69 \times 10^{8}\left(N^{2}\right)$ & $1.35 \times 10^{4}(N)$ \\
\hline
\end{tabular}
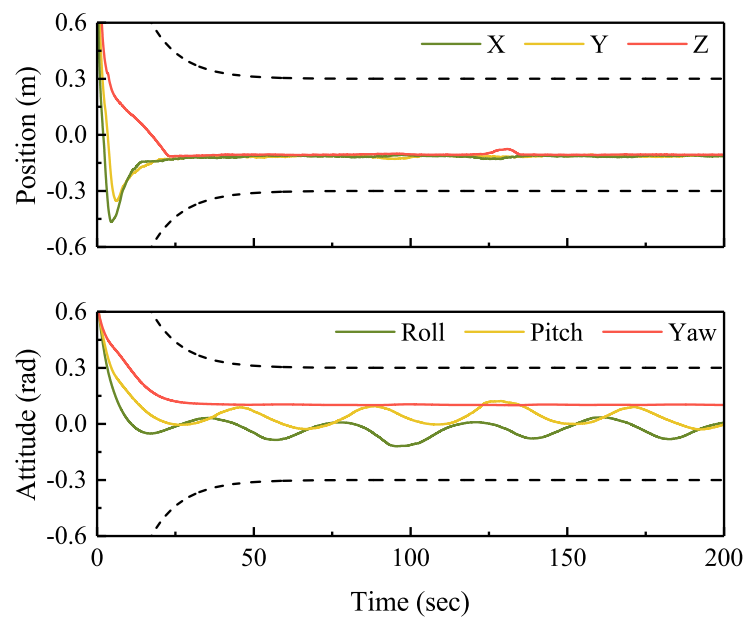

(a) Tracking error
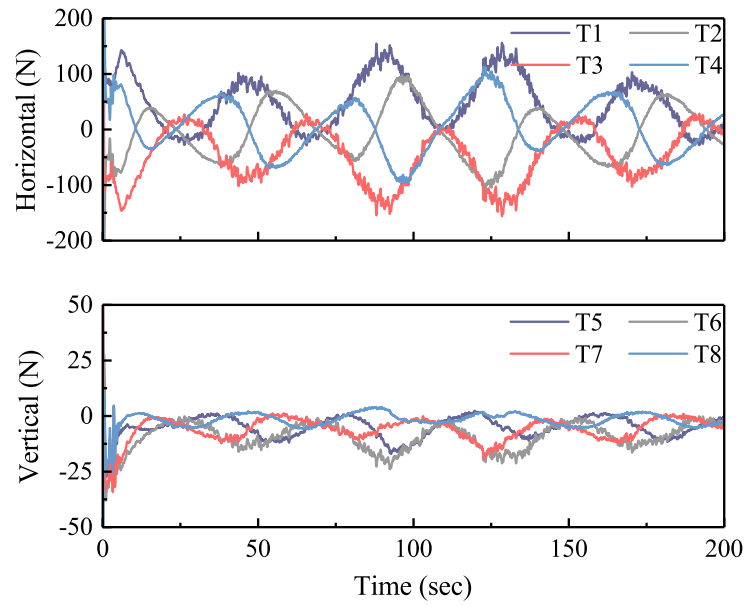

(b) Control input

Fig. 3 Tracking results based on the proposed observerbased region tracking control strategy without measurement noise

\section{Conclusions}

This paper presents an observer-based region tracking control strategy for underwater vehicles without veloc-

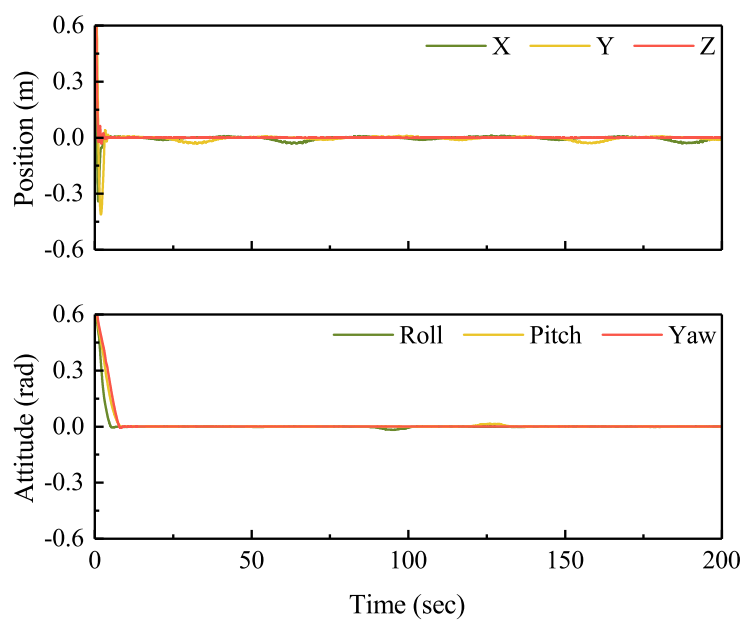

(a) Tracking error
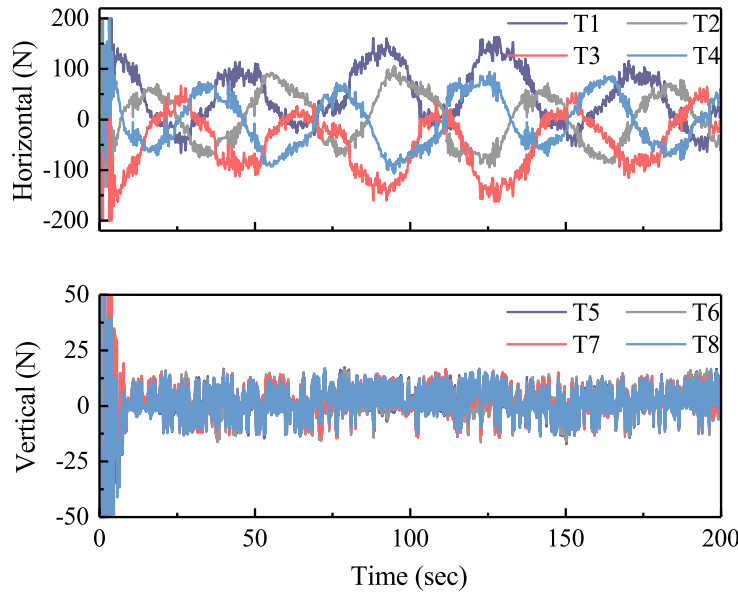

(b) Control input

Fig. 4 Tracking results based on the conventional backstepping control scheme without measurement noise

ity measurement. To estimate the unmeasured velocity of the vehicle, a Nussbaum state observer is developed and it is verified that the estimation error is uniformly ultimately bounded. Then, according to the estimated velocity, a region tracking control scheme to- 

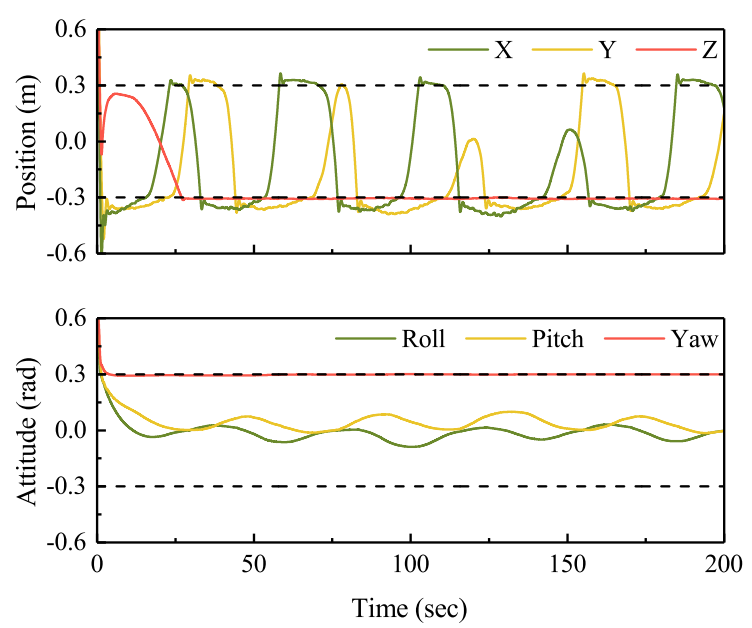

(a) Tracking error
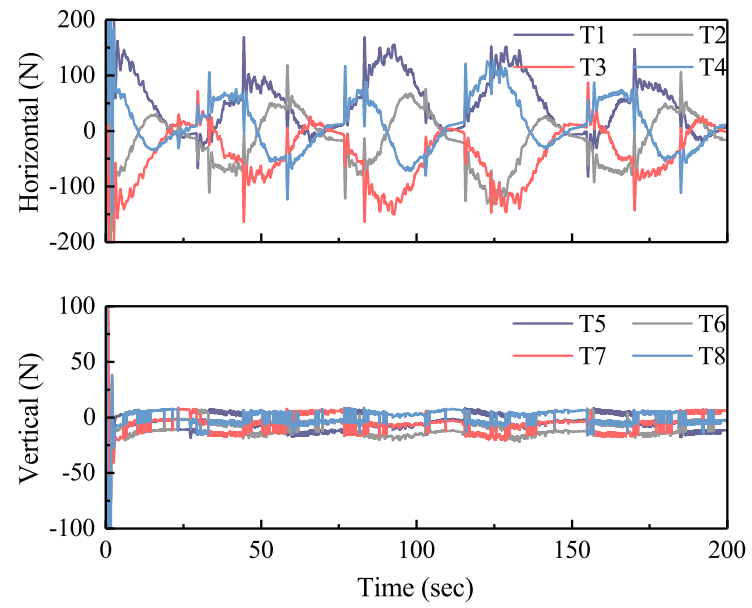

(b) Control input

Fig. 5 Tracking results of the conventional region tracking control scheme based on high-gain observer without measurement noise

gether with a new error transformation is designed for the vehicle in the framework of backstepping technique. The uniform ultimate boundedness of the tracking errors are proved based on Lyapunov theory. Simulation results on a fully-actuated underwater vehicle also confirm that the tracking errors are always kept within the prescribed boundaries based on the proposed observerbased region tracking control strategy without velocity measurement, even in presence of measurement noise, and the corresponding control signals are much smoother than the traditional backstepping control scheme and conventional region tracking scheme.

Acknowledgements This project is supported by National Natural Science Foundation of China under Grant 51839004, Natural Science Foundation of Zhejiang Province under Grant No. LQ20E090009, and Fund from Science and Technology on Underwater Vehicle Technology under Grant 6142215190308,
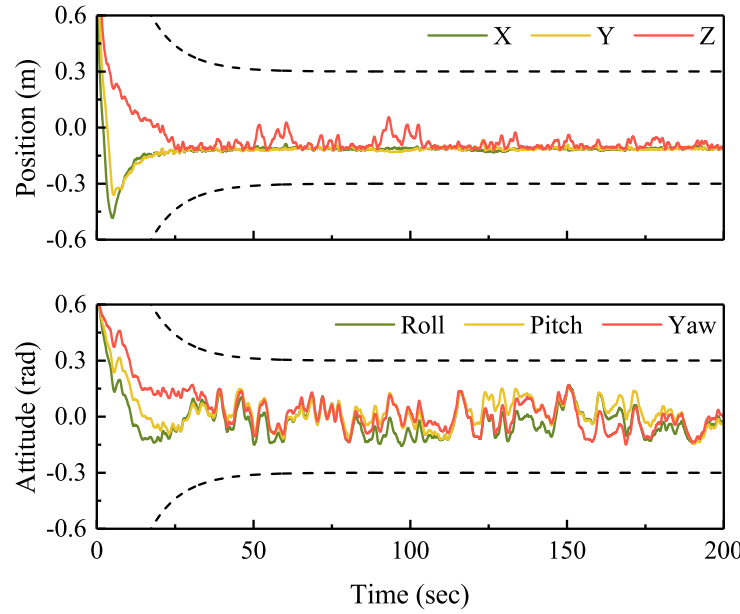

(a) Tracking error
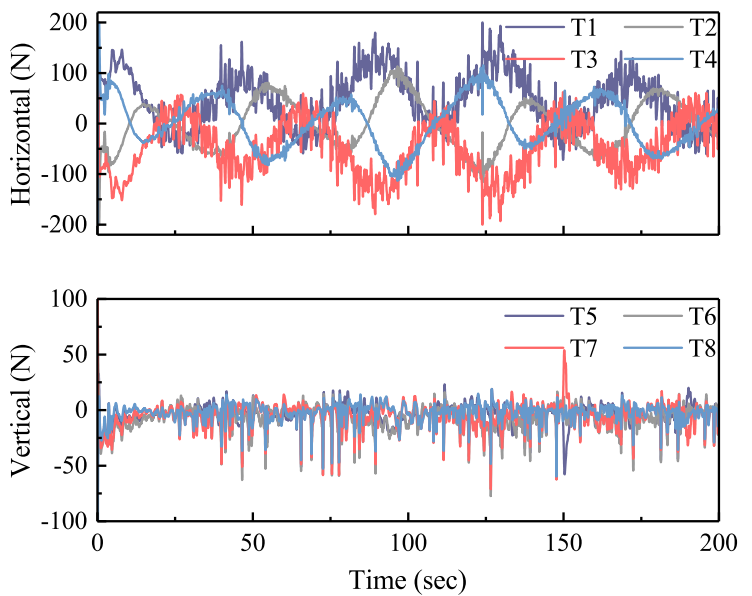

(b) Control input

Fig. 6 Tracking results based on the proposed observerbased region tracking control strategy with measurement noise

and the fundamental research funds for the central universities under Grant 3072021CFJ0703..

\section{Conflict of interest}

The authors declare that they have no conflict of interest.

\section{Data availability statement}

The datasets generated during and/or analysed during the current study are available from the corresponding author on reasonable request. 

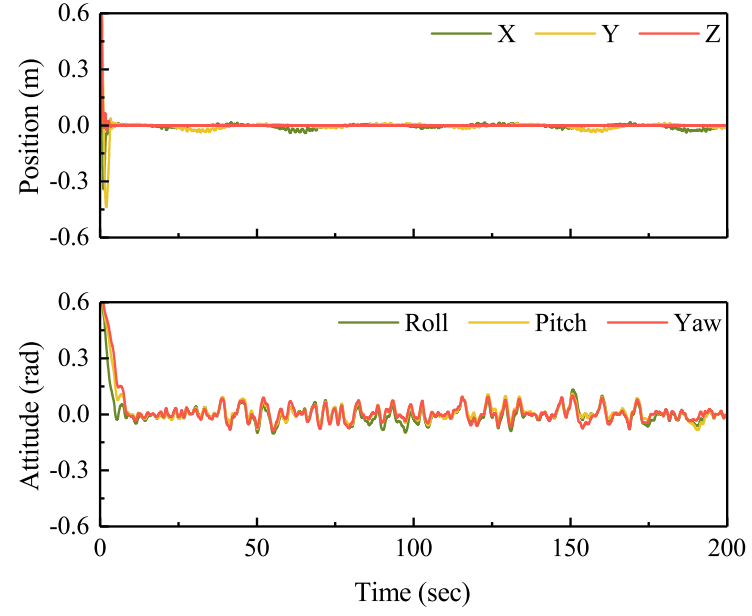

(a) Tracking error
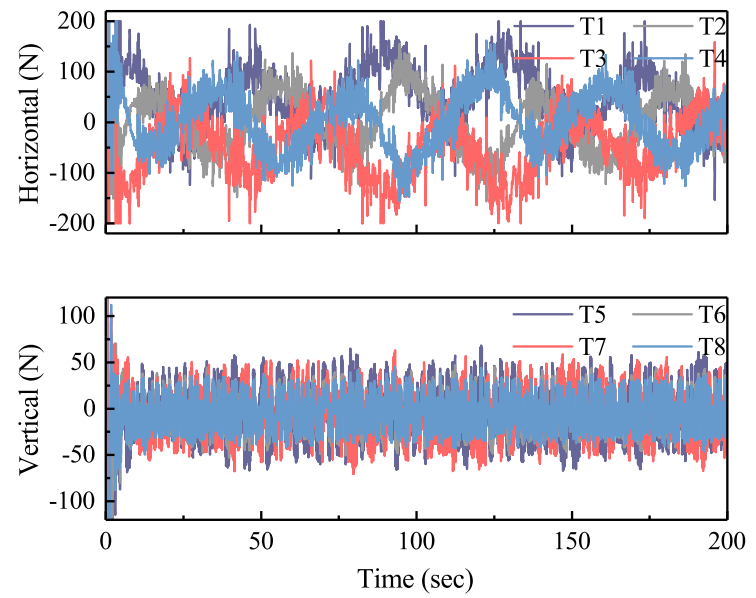

(b) Control input

Fig. 7 Tracking results based on the conventional backstepping control scheme with measurement noise

\section{References}

1. Zhang, Y., Ryan, J.P., Kieft, B., Hobson, B.W., McEwen, R.S., Godin, M.A., Harvey, J.B., Barone, B., Bellingham, J.G., Birch, J.M., Scholin, C.A., Chavez, F.P.: Targeted sampling by autonomous underwater vehicles. Frontiers in Marine Science 6 (2019). DOI 10.3389/fmars.2019. 00415

2. Matsuda, T., Maki, T., Masuda, K., Sakamaki, T.: Resident autonomous underwater vehicle: Underwater system for prolonged and continuous monitoring based at a seafloor station. Robotics and Autonomous Systems 120 (2019). DOI 10.1016/j.robot.2019.07.001

3. Duan, K., Fong, S., Chen, C.L.P.: Multilayer neural networks-based control of underwater vehicles with uncertain dynamics and disturbances. Nonlinear Dynamics (2020). DOI 10.1007/s11071-020-05720-5

4. Cui, S.W., Wang, Y., Wang, S., Wang, R., Wang, W., Tan, M.: Real-time perception and positioning for creature picking of an underwater vehicle. Ieee Transactions on Vehicular Technology 69(4), 3783-3792 (2020). DOI $10.1109 /$ tvt.2020.2973656
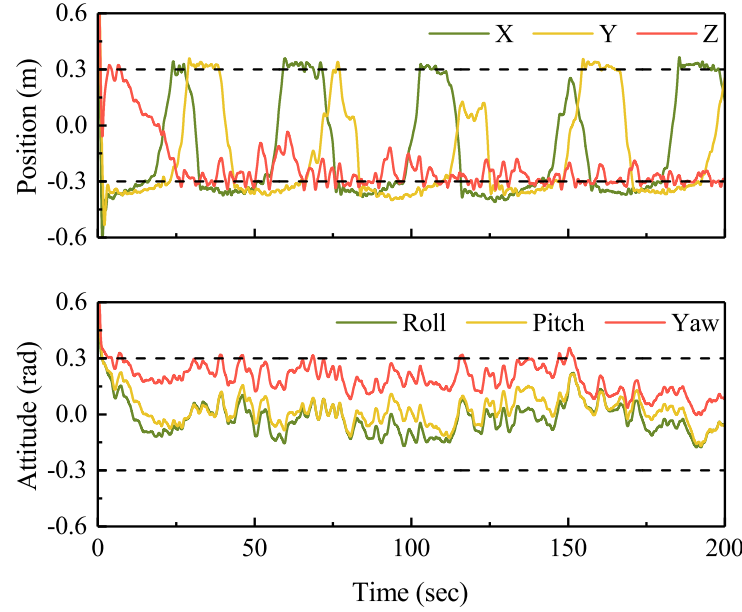

(a) Tracking error
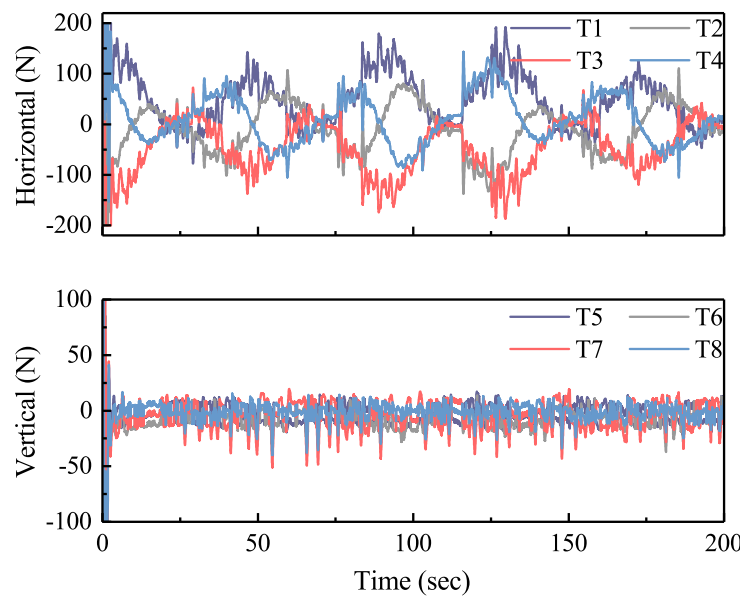

(b) Control input

Fig. 8 Tracking results of the conventional region tracking control scheme based on high-gain observer with measurement noise

5. Shen, C., Shi, Y., Buckham, B.: Integrated path planning and tracking control of an auv: A unified receding horizon optimization approach. IEEE/ASME Transactions on Mechatronics 22(3), 1163-1173 (2017). DOI 10.1109/tmech.2016.2612689

6. Martin, S.C., Whitcomb, L.L.: Nonlinear model-based tracking control of underwater vehicles with three degreeof-freedom fully coupled dynamical plant models: Theory and experimental evaluation. IEEE Transactions on Control Systems Technology 26(2), 404-414 (2018). DOI 10.1109/tcst.2017.2665974

7. Qiao, L., Zhang, W.: Adaptive second-order fast nonsingular terminal sliding mode tracking control for fully actuated autonomous underwater vehicles. IEEE Journal of Oceanic Engineering 44(2), 363-385 (2019). DOI 10.1109/joe.2018.2809018

8. Xu, R., Tang, G., Han, L., Xie, D.: Trajectory tracking control for a cmg-based underwater vehicle with input saturation in 3d space. Ocean Engineering 173, 587-598 (2019). DOI 10.1016/j.oceaneng.2018.12.069

9. Peng, Z., Wang, J.: Output-feedback path-following control of autonomous underwater vehicles based on an ex- 
tended state observer and projection neural networks. IEEE Transactions on Systems, Man, and Cybernetics: Systems 48(4), 535-544 (2018). DOI 10.1109/tsmc.2017. 2697447

10. Guerrero, J., Torres, J., Creuze, V., Chemori, A.: Trajectory tracking for autonomous underwater vehicle: An adaptive approach. Ocean Engineering 172, 511-522 (2019). DOI 10.1016/j.oceaneng.2018.12.027

11. Yu, C., Xiang, X., Wilson, P.A., Zhang, Q.: Guidanceerror-based robust fuzzy adaptive control for bottom following of a flight-style auv with saturated actuator dynamics. IEEE Trans Cybern 50(5), 1887-1899 (2020). DOI 10.1109/TCYB.2018.2890582

12. Li, X., Hou, S.P., Cheah, C.C.: Adaptive region tracking control for autonomous underwater vehicle. In: 2010 11th International Conference on Control Automation Robotics \& Vision (ICARCV), pp. 2129-2134 (2010). DOI 10.1109/ICARCV.2010.5707863

13. Ismail, Z.H., Dunnigan, M.W.: Tracking control scheme for an underwater vehicle-manipulator system with single and multiple sub-regions and sub-task objectives. IET Control Theory \& Applications 5(5), 721-735 (2011). DOI 10.1049/iet-cta.2010.0174

14. Zhang, M., Chu, Z.: Adaptive region tracking control for autonomous underwater vehicle. Journal of Mechanical Engineering 50(19), 50-57 (2014). DOI 10.3901/jme. 2014.19.050

15. Mukherjee, K., Kat, I.N., Bhatt, R.K.P.: Region tracking based control of an autonomous underwater vehicle with input delay. Ocean Engineering 99, 107-114 (2015). DOI DOI10.1016/j.oceaneng.2015.02.006

16. Chu, Z., Zhu, D.: Fault-tolerant control of autonomous underwater vehicle based on adaptive region tracking. Journal of Shandong University (Engineering Science) 47(5), 57-63 (2017)

17. Zhang, M.J., Liu, X., Wang, F.: Backstepping based adaptive region tracking fault tolerant control for autonomous underwater vehicles. Journal of Navigation 70(1), 184-204 (2017). DOI 10.1017/S0373463316000370

18. Bechlioulis, C.P., Rovithakis, G.A.: A low-complexity global approximation-free control scheme with prescribed performance for unknown pure feedback systems. Automatica 50(4), 1217-1226 (2014). DOI $10.1016 / \mathrm{j}$. automatica.2014.02.020

19. Zhang, J.X., Yang, G.H.: Prescribed performance faulttolerant control of uncertain nonlinear systems with unknown control directions. IEEE Transactions on Automatic Control 62(12), 6529-6535 (2017). DOI 10.1109/ Tac.2017.2705033

20. Shao, X., Hu, Q., Shi, Y., Jiang, B.: Fault-tolerant prescribed performance attitude tracking control for spacecraft under input saturation. IEEE Transactions on Control Systems Technology 28(2), 574-582 (2020). DOI $10.1109 /$ tcst.2018.2875426

21. Liu, X., Zhang, M., Wang, S.: Adaptive region tracking control with prescribed transient performance for autonomous underwater vehicle with thruster fault. Ocean Engineering 196 (2020). DOI 10.1016/j.oceaneng.2019. 106804

22. Chu, Z., Zhu, D., Yang, S.X.: Observer-based adaptive neural network trajectory tracking control for remotely operated vehicle. IEEE Transactions on Neural Networks and Learning Systems 28(7), 1633-1645 (2017). DOI 10.1109/TNNLS.2016.2544786

23. Liu, X., Zhang, M., Wang, Y., Rogers, E.: Design and experimental validation of an adaptive sliding mode observer-based fault-tolerant control for underwater vehicles. IEEE Transactions on Control Systems Technology 27(6), 2655-2662 (2019). DOI 10.1109/tcst.2018.2870829

24. Kinsey, J.C., Yang, Q.J., Howland, J.C.: Nonlinear dynamic model-based state estimators for underwater navigation of remotely operated vehicles. IEEE Transactions on Control Systems Technology 22(5), 1845-1854 (2014). DOI 10.1109/Tcst.2013.2293958

25. Liu, S., Liu, Y., Wang, N.: Nonlinear disturbance observer-based backstepping finite-time sliding mode tracking control of underwater vehicles with system uncertainties and external disturbances. Nonlinear Dynamics 88(1), 465-476 (2017). DOI 10.1007/ s11071-016-3253-8

26. Fernandes, D.D., Sorensen, A.J., Pettersen, K.Y., Donha, D.C.: Output feedback motion control system for observation class rovs based on a high-gain state observer: Theoretical and experimental results. Control Engineering Practice 39, 90-102 (2015). DOI 10.1016/j.conengprac. 2014.12.005

27. Duan, K.R., Fong, S., Chen, C.L.P.: Fuzzy observerbased tracking control of an underactuated underwater vehicle with linear velocity estimation. IET Control Theory and Applications 14(4), 584-593 (2020). DOI 10.1049/iet-cta.2019.0604

28. Chen, M., Yu, J.: Adaptive dynamic surface control of nsvs with input saturation using a disturbance observer. Chinese Journal of Aeronautics 28(3), 853-864 (2015). DOI 10.1016/j.cja.2015.04.020

29. Chen, M., Shao, S.Y., Jiang, B.: Adaptive neural control of uncertain nonlinear systems using disturbance observer. IEEE Trans Cybern 47(10), 3110-3123 (2017). DOI 10.1109/TCYB.2017.2667680

30. Fossen, T.I.: Handbook of marine craft hydrodynamics and motion control. John Wiley \& Sons, New York (2011)

31. Song, Y., Huang, X., Wen, C.: Robust adaptive faulttolerant pid control of mimo nonlinear systems with unknown control direction. IEEE Transactions on Industrial Electronics 64(6), 4876-4884 (2017). DOI 10.1109/tie.2017.2669891

32. Hu, Q., Shao, X., Guo, L.: Adaptive fault-tolerant attitude tracking control of spacecraft with prescribed performance. IEEE/ASME Transactions on Mechatronics 23(1), 331-341 (2018). DOI 10.1109/TMECH.2017. 2775626

33. Chu, Z., Meng, F., Zhu, D., Luo, C.: Fault reconstruction using a terminal sliding mode observer for a class of second-order mimo uncertain nonlinear systems. ISA Trans 97, 67-75 (2020). DOI 10.1016/j.isatra.2019.07. 024

34. Aghababa, M.P., Moradi, S.: Robust adaptive dynamic surface back-stepping tracking control of high-order strict-feedback nonlinear systems via disturbance observer approach. International Journal of Control pp. 1-17 (2020). DOI 10.1080/00207179.2020.1712478

35. Tan, C.P., Yu, X.H., Man, Z.H.: Terminal sliding mode observers for a class of nonlinear systems. Automatica 46(8), 1401-1404 (2010). DOI 10.1016/j.automatica. 2010.05.010

36. Tee, K.P., Ge, S.S., Tay, E.H.: Barrier lyapunov functions for the control of output-constrained nonlinear systems. Automatica 45(4), 918-927 (2009). DOI 10.1016/j.automatica.2008.11.017

37. Li, H., Zhao, S., He, W., Lu, R.: Adaptive finite-time tracking control of full state constrained nonlinear systems with dead-zone. Automatica 100, 99-107 (2019). DOI 10.1016/j.automatica.2018.10.030 
38. Gao, J., An, X., Proctor, A., Bradley, C.: Sliding mode adaptive neural network control for hybrid visual servoing of underwater vehicles. Ocean Engineering 142, 666-675 (2017). DOI 10.1016/j.oceaneng.2017.07.015

39. Liu, G., Hou, Z.: Rbfnn-based adaptive iterative learning fault-tolerant control for subway trains with actuator faults and speed constraint. IEEE Transactions on Systems, Man, and Cybernetics: Systems pp. 1-15 (2019). DOI 10.1109/tsmc.2019.2957299

40. Song, Y., Zhou, S.: Neuroadaptive control with given performance specifications for mimo strict-feedback systems under nonsmooth actuation and output constraints. IEEE Trans Neural Netw Learn Syst 29(9), 4414-4425 (2018). DOI 10.1109/TNNLS.2017.2766123

41. Du, J., Hu, X., Krstić, M., Sun, Y.: Robust dynamic positioning of ships with disturbances under input saturation. Automatica 73, 207-214 (2016). DOI 10.1016/j. automatica.2016.06.020

42. Qiao, L., Zhang, W.D.: Double-loop integral terminal sliding mode tracking control for uuvs with adaptive dynamic compensation of uncertainties and disturbances. IEEE Journal of Oceanic Engineering 44(1), 2953 (2019). DOI 10.1109/joe.2017.2777638 